\title{
Spherical f-Tilings by Scalene Triangles and Isosceles Trapezoids III
}

\author{
Catarina P. Avelino * Altino F. Santos ${ }^{\dagger}$ \\ Department of Mathematics \\ UTAD, 5001 - 801 Vila Real, Portugal
}

Submitted: Jun 23, 2009; Accepted: Jul 13, 2009; Published: Jul 24, 2009

Mathematics Subject Classification: 52C20, 52B05, 20B35

\begin{abstract}
The study of the dihedral f-tilings of the sphere $S^{2}$ whose prototiles are a scalene triangle and an isosceles trapezoid was initiated in $[7,8]$. In this paper we complete this classification presenting the study of all dihedral spherical f-tilings by scalene triangles and isosceles trapezoids in the remaining case of adjacency. A list containing all the f-tilings obtained in this paper is presented in Table 1. It is composed by isolated tilings as well as discrete and continuous families of tilings. The combinatorial structure is also achieved.
\end{abstract}

Keywords: dihedral f-tilings, combinatorial properties, spherical trigonometry

\section{Introduction}

Let $S^{2}$ be the Euclidean sphere of radius 1. By a dihedral folding tiling (f-tiling, for short) of the sphere $S^{2}$ whose prototiles are a spherical isosceles trapezoid, $Q$, and a spherical triangle, $T$, we mean a polygonal subdivision $\tau$ of $S^{2}$ such that each cell (tile) of $\tau$ is congruent to $Q$ or $T$ and the vertices of $\tau$ satisfy the angle-folding relation, i.e., each vertex of $\tau$ is of even valency $2 n, n \geq 2$, and the sums of alternate angles are equal; that is,

$$
\sum_{i=1}^{n} \theta_{2 i}=\sum_{i=1}^{n} \theta_{2 i-1}=\pi
$$

where the angles $\theta_{i}$ around any vertex of $\tau$ are ordered cyclically.

\footnotetext{
*(cavelino@utad.pt)

$\dagger($ afolgado@utad.pt)
}

Supported partially by the Research Unit CM-UTAD of University of Trás-os-Montes e Alto Douro, through the Foundation for Science and Technology (FCT). 
Folding tilings are intrinsically related to the theory of isometric foldings on Riemannian manifolds. In fact, the set of singularities of any spherical isometric folding corresponds to a folding tiling of the sphere, see [9] for the foundations of this subject.

The study of dihedral f-tilings of the sphere started in 2004 [1, 2, 3], where the classification of all dihedral f-tilings by spherical parallelograms and spherical triangles was obtained. Later on, in [5], the classification of all dihedral f-tilings of the sphere by triangles and $r$-sided regular polygons $(r \geq 5)$ was achieved. In a subsequent paper [4], is presented the study of all dihedral spherical f-tilings whose prototiles are an equilateral triangle and an isosceles triangle. Robert Dawson and B. Doyle have also been interested in special classes of spherical tilings, see $[10,11]$ for instance.

In this paper we shall discuss dihedral f-tilings by spherical scalene triangles, $T$, and spherical isosceles trapezoids, $Q$, with a certain adjacency pattern. We present in Table 1 a list containing all the $\mathrm{f}$-tilings obtained in this paper. We shall denote by $\Omega(Q, T)$ the set, up to an isomorphism, of all dihedral f-tilings of $S^{2}$ whose prototiles are $Q$ and $T$.

From now on $Q$ is a spherical isosceles trapezoid of internal angles $\alpha_{1}$ and $\alpha_{2}\left(\alpha_{1}>\alpha_{2}\right)$ and edge lengths $a, b$ and $c(b>c)$, and $T$ is a spherical scalene triangle of internal angles $\beta, \gamma$ and $\delta(\beta>\gamma>\delta$ ), with edge lengths $d$ (opposite to $\beta$ ), $e$ (opposite to $\gamma$ ) and $f$ (opposite to $\delta$ ), see Figure 1 .
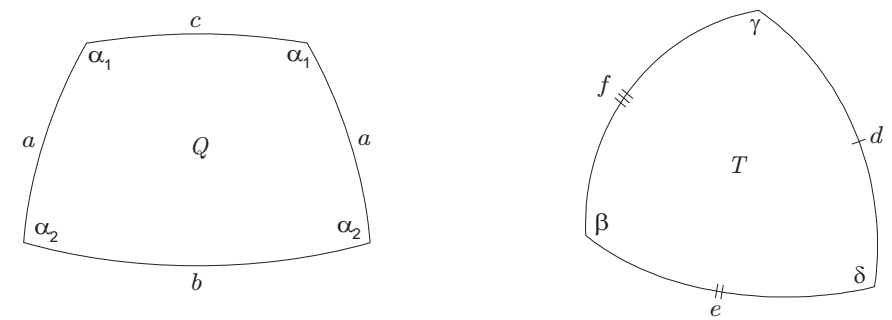

Figure 1: Prototiles: a spherical isosceles trapezoid and a spherical scalene triangle

It follows immediately that

$$
\beta+\gamma+\delta>\pi \text { and } \alpha_{1}+\alpha_{2}>\pi, \text { with } \alpha_{1}>\frac{\pi}{2} .
$$

In order to get any dihedral f-tiling $\tau \in \Omega(Q, T)$, we find useful to start by considering one of its local configurations, beginning with a common vertex to two tiles of $\tau$ in adjacent positions.

In the diagrams that follows it is convenient to label the tiles according to the following procedures:

(i) We begin the configuration of a tiling $\tau \in \Omega(Q, T)$ with an isosceles trapezoid, labelled by 1 ; then we label with $1^{\prime}$ an isosceles trapezoid or a scalene triangle adjacent to tile 1 and sharing the side of length $c$;

(ii) For $j \geq 2$, the location of tile $j$ can be deduced from the configuration of tiles $\left(1,1^{\prime}, 2,3, \ldots, j-1\right)$ and from the hypothesis that the configuration is part of a complete f-tiling (except in the cases indicated). 


\section{Dihedral Spherical f-Tilings by Scalene Triangles and Isosceles Trapezoids}

Any element of $\Omega(Q, T)$ has at least two cells such that they are in adjacent positions and in one of the situations illustrated in Figure 2. The cases of adjacency $I-I V$ were
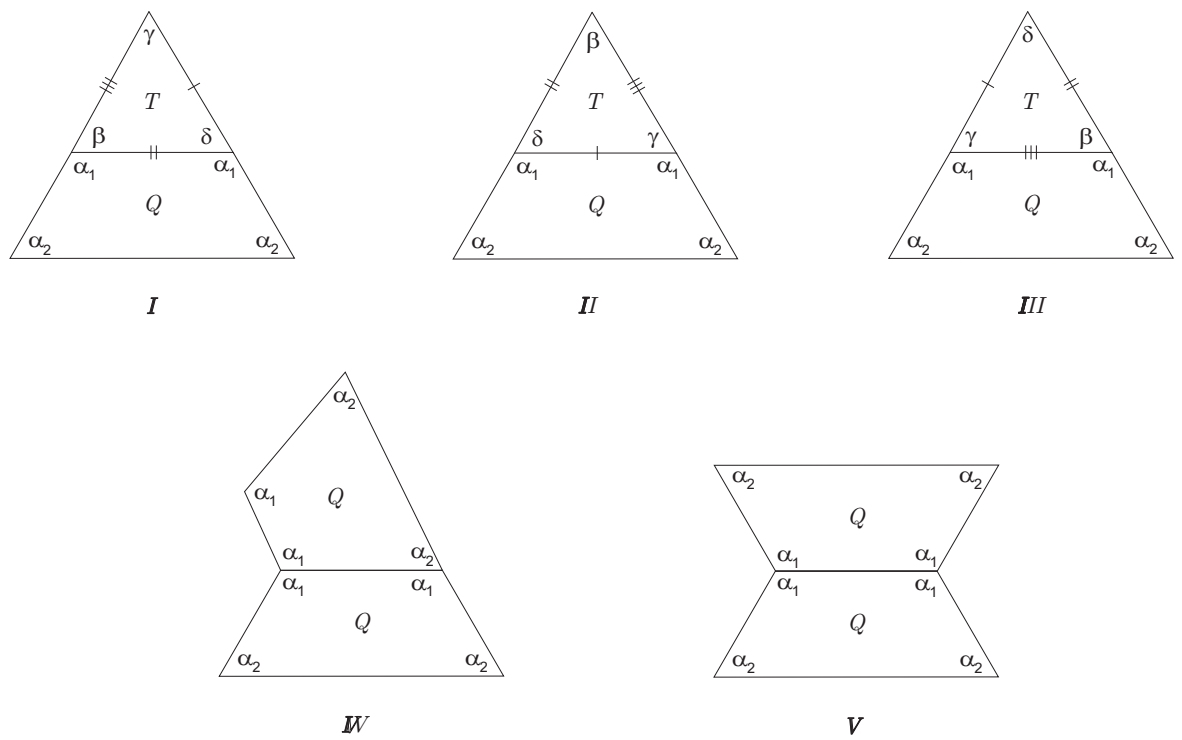

Figure 2: Distinct cases of adjacency

analyzed in $[7,8]$. In this paper we complete the study of all dihedral spherical f-tilings by scalene triangles and isosceles trapezoids through the analysis of the case of adjacency $V$.

In the following results we will use the fact that we cannot have a spherical trapezoid adjacent to a spherical triangle and sharing the side of length $c$, and also that we cannot have two trapezoids sharing the sides of length $a$ and $c$, respectively. These situations have already been studied before $([7,8])$ and do not give rise to any f-tiling when there are two trapezoids sharing the side of length $c$. Consequently, a trapezoid must always have an adjacent trapezoid sharing the side of length $c$; and, on the other hand, we cannot have two trapezoids sharing the sides of length $a$ and $b$ (nor $b$ and $c$ ).

Suppose that any element of $\Omega(Q, T)$ has at least two cells such that they are in adjacent positions as illustrated in Figure 3. With the labelling of this figure, we have necessarily

$$
\theta_{1}=\beta, \quad \theta_{1}=\delta \quad \text { or } \quad \theta_{1}=\gamma
$$

These three distinct cases will be now analyzed separately in the following propositions.

Proposition 2.1 With the above terminology, if $\theta_{1}=\beta$, then $\Omega(Q, T)=\emptyset$.

Proof. Suppose that we have two cells in adjacent positions as illustrated in Figure 4. 


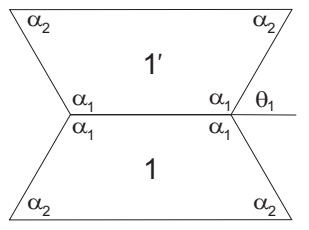

Figure 3: Local configuration

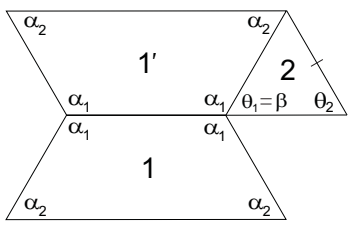

Figure 4: Local configuration

As $\alpha_{1}>\alpha_{2}>\beta>\gamma>\delta$ and $\beta+\gamma+\delta>\pi$, we have necessarily $\alpha_{1}+\beta=\pi$. With the labelling used in Figure 4, we have $\theta_{2}=\gamma$ or $\theta_{2}=\delta$.

If $\theta_{2}=\gamma$ (Figure 5(a)), then tile 3 comes in a unique way and we must have $\theta_{3}=\delta$. In fact, since $v$ has valency greater than four $\left(\delta+\rho<\pi, \forall \rho \in\left\{\alpha_{1}, \alpha_{2}, \beta, \gamma, \delta\right\}\right)$, we have $\alpha_{2}+\alpha_{2}+\rho>\alpha_{2}+\gamma+\rho>\beta+\gamma+\delta>\pi, \forall \rho \in\left\{\alpha_{1}, \alpha_{2}, \beta, \gamma, \delta\right\}$.

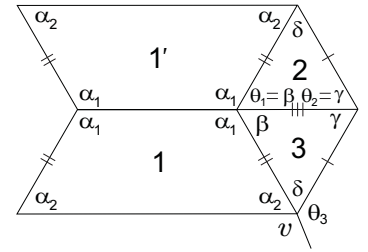

(a)

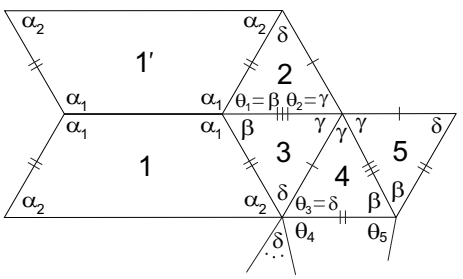

(b)

Figure 5: Local configurations

Therefore, $\theta_{3}=\delta$ and also $\alpha_{2}+k \delta=\pi$, for some $k \geq 2$, and we get the local configuration illustrated in Figure 5(b). Now, we have $\theta_{4}=\alpha_{2}$ and $\theta_{5}=\alpha_{1}$. Note that $\theta_{4}$ cannot be $\delta$, since $\theta_{4}=\delta$ implies $\theta_{5}=\beta$ and $\beta+\beta<\pi<\beta+\beta+\rho, \forall \rho \in\left\{\alpha_{1}, \alpha_{2}, \beta, \gamma, \delta\right\}$. Taking into account the angles and edge lengths, $\theta_{6}=\beta$ or $\theta_{6}=\gamma$ (Figure 6(a)). But $\alpha_{2}+\theta_{6}<\pi<\alpha_{2}+\theta_{6}+\rho, \forall \rho \in\left\{\alpha_{1}, \alpha_{2}, \beta, \gamma, \delta\right\}$, which is a contradiction.

If $\theta_{2}=\delta$ (Figure 4), then we obtain the configuration illustrated in Figure 6(b). Note that, using similar argumentation to the one used before, we must have $\theta_{3}=\delta$. Now, taking into account the relation between angles and edge lengths, $\theta_{4}$ must be $\delta$, and so we get $\alpha_{2}+k \delta=\pi=\gamma+k \delta$, for some $k \geq 2$, which is a contradiction (note that $\alpha_{2}>\gamma$ ).

Proposition 2.2 If $\theta_{1}=\delta$ and $\alpha_{1}+\delta<\pi$, then $\Omega(Q, T)$ is composed by a single tiling, denoted by $\mathcal{N}$, where $\alpha_{1}=\frac{3 \pi}{5}, \alpha_{2}=\beta=\frac{\pi}{2}, \gamma=\frac{\pi}{3}$ and $\delta=\frac{\pi}{5}$. The angles around vertices are positioned as illustrated in Figure \%. For a planar representation see Figure 15. Its $3 D$ representation is given in Figure 16. 


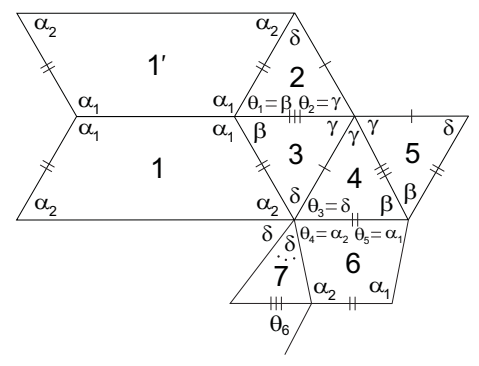

(a)

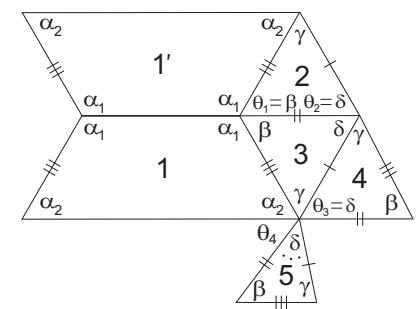

(b)

Figure 6: Local configurations

\begin{tabular}{|c|c|c|c|c|c|}
\hline \begin{tabular}{l|l}
$\alpha_{1}$ & $\alpha_{1}$
\end{tabular} & $\beta$ & $\beta$ & $\alpha_{2}$ & $\alpha_{2}$ & $\gamma>\gamma / \gamma$ \\
\hline$\delta / \delta \delta \delta$ & $\beta$ & $\beta$ & $\beta$ & $\beta$ & $\gamma / \gamma \gamma$ \\
\hline
\end{tabular}

Figure 7: Distinct classes of congruent vertices

Proof. Suppose that we have two cells in adjacent positions as illustrated in Figure 3 and consider $\theta_{1}=\delta$, with $\alpha_{1}+\delta<\pi$. With the labelling of Figure $8(\mathrm{a})$, we have

$$
\theta_{2}=\gamma \quad \text { or } \quad \theta_{2}=\beta
$$

1. Suppose firstly that $\theta_{2}=\gamma$. We consider separately the cases $\alpha_{1}<\beta$ and $\alpha_{1} \geq \beta$.

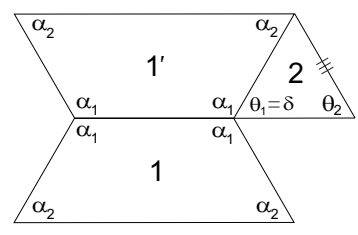

(a)

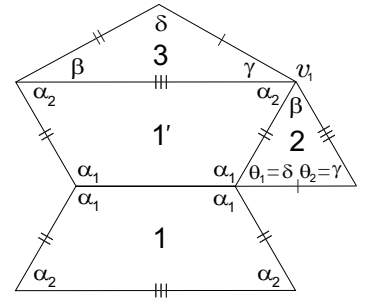

(b)

Figure 8: Local configurations

1.1 If $\alpha_{1}<\beta$, we have $\beta+\gamma=\pi$ or $\beta+k \delta=\pi$, for some $k \geq 1$.

As we can observe in Figure $8(\mathrm{~b})$, the case $\beta+\gamma=\pi$ leads to a contradiction since there is no way to avoid an incompatibility at vertex $v_{1}$.

If $\beta+\delta=\pi$ (Figure $9(\mathrm{a})$ ), we also reach a contradiction at vertex $v_{2}$. Note that $\theta_{3}$ must be $\beta$, otherwise there is no way to satisfy the angle-folding relation around vertex $v_{1}$.

Suppose finally that $\beta+k \delta=\pi$, for some $k \geq 2$. At vertex $v_{1}$ (Figure $9(\mathrm{~b})$ ), we obtain $\beta+k \delta=\pi=\alpha_{2}+\sum_{i=1}^{k-1} \rho_{i}+\beta$, with $\rho_{i} \in\left\{\alpha_{2}, \delta\right\}, i=1,2, \ldots, k-1$ (see edge lengths), which is a contradiction (note that we have considered $b=d$, see Figure 1 , as the case $b=e$ lies in the previous one). 


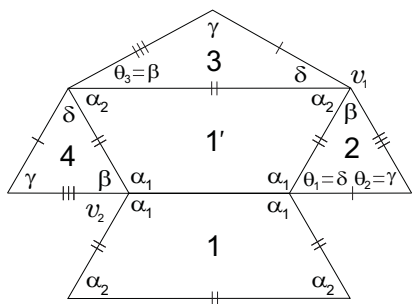

(a)

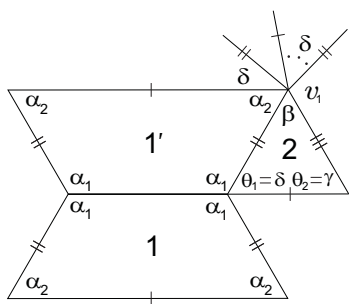

(b)

Figure 9: Local configurations

1.2 If $\alpha_{1} \geq \beta$, then $\alpha_{1}+k \delta=\pi$, for some $k \geq 2$, and we obtain the local configuration given in Figure 10(a). Now, if tile 10 is a triangle, then $\theta_{3}$ must be $\beta, \gamma$ or $\delta$. In all cases we reach a contradiction at vertex $v$, see Figure 10(b), Figure 11(a) and Figure 11(b), respectively.

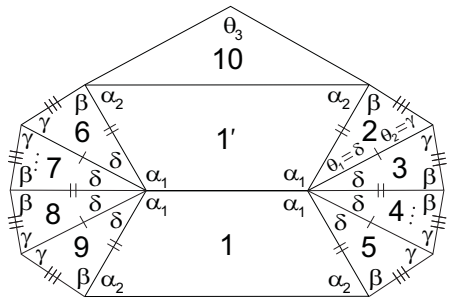

(a)

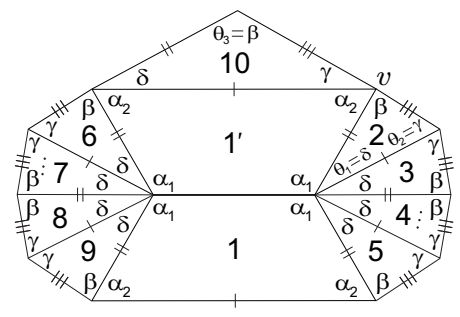

(b)

Figure 10: Local configurations

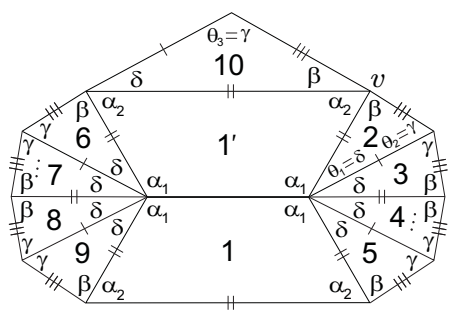

(a)

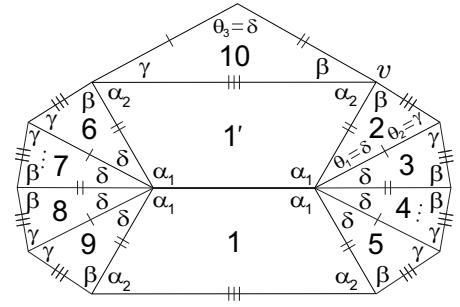

(b)

Figure 11: Local configurations

Therefore, tile 10 must be a trapezoid and, using analogous arguments, we get the local configuration illustrated in Figure 12(a). Now, at vertex $v_{1}$, we have $\alpha_{2}+\beta<\pi$ or $\alpha_{2}+\beta=\pi$.

1.2.1 Suppose firstly that $\alpha_{2}+\beta<\pi$. Therefore $\alpha_{1}>\beta>\gamma>\alpha_{2}>\delta$. If $v_{1}$ has valency six, then $\alpha_{2}+\beta+\alpha_{2}=\pi$ (see edge lengths) and we reach a contradiction at vertex $v_{2}$ in Figure $12(\mathrm{~b})$. On the other hand, if $v_{1}$ has valency greater than six, we 


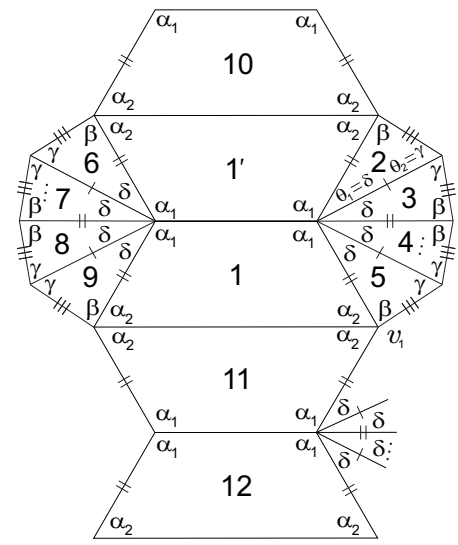

(a)

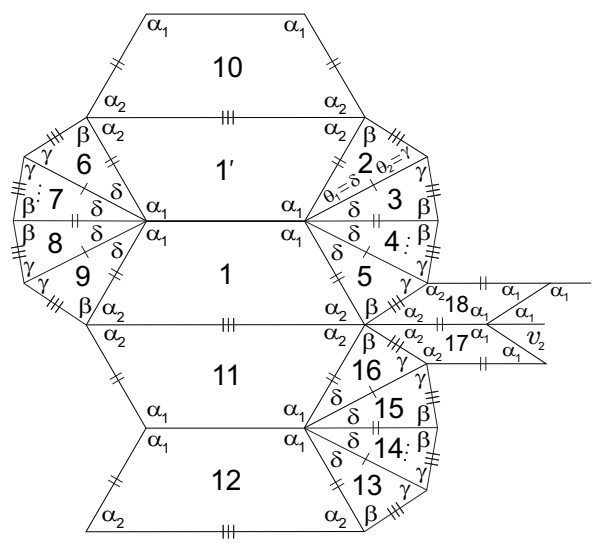

(b)

Figure 12: Local configurations

reach a contradiction at vertex $v_{3}$ in Figure $13(\mathrm{a})$, since there is no way to satisfy the angle-folding relation around this vertex.

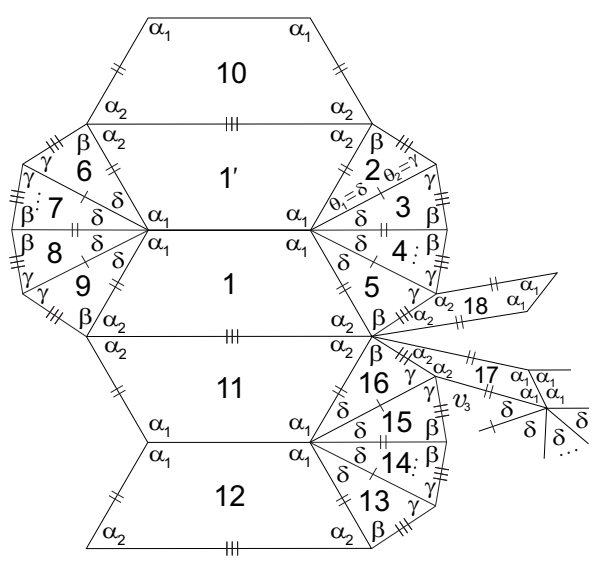

(a)

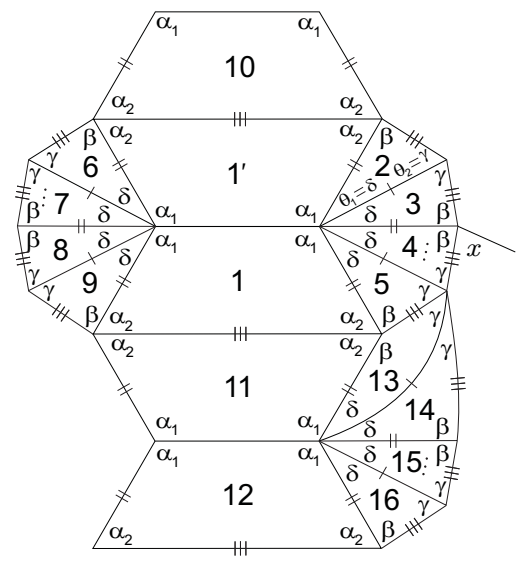

(b)

Figure 13: Local configurations

1.2.2 Suppose now that $\alpha_{2}+\beta=\pi$. Then, the configuration illustrated in Figure 12(a) is extended in a unique way to the one given in Figure 13(b). With the labelling of this figure, we have $x=\alpha_{2}$ or $x=\beta$.

If $x=\alpha_{2}$, we obtain the configuration given in Figure 14. At vertex $v_{4}$, one of the alternating angle sums must contain the sequence $(\ldots, \beta, \gamma, \gamma, \ldots)$. But $\beta+\gamma+\gamma>\pi$, which is an impossibility.

If $x=\beta$, then $\alpha_{2}=\beta=\frac{\pi}{2}$ and consequently $\gamma+\delta>\frac{\pi}{2}$. It follows that a vertex surrounded by six angles $\gamma$ takes place, i.e., $\gamma=\frac{\pi}{3}$. And so $\delta>\frac{\pi}{6}$, since $\beta+\gamma+\delta>\pi$. The construction of the planar configuration is unique (Figure 15) and leads to a vertex surrounded by only angles $\delta$. Therefore $\delta=\frac{\pi}{4}$ or $\delta=\frac{\pi}{5}$. However, if $\delta=\frac{\pi}{4}$, then 


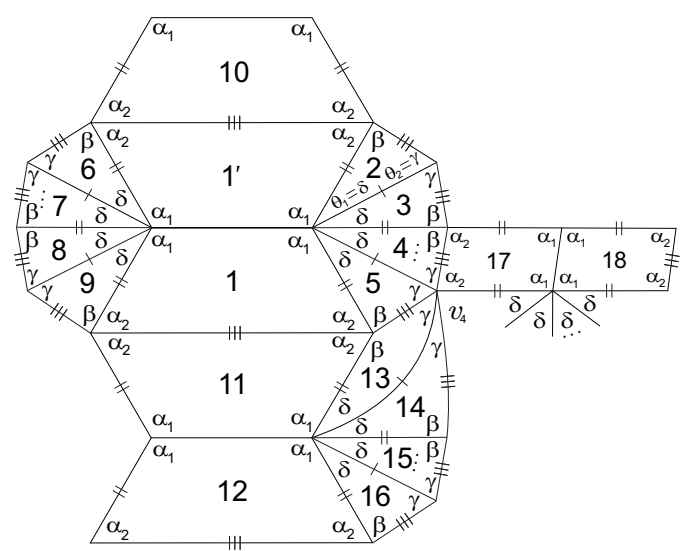

Figure 14: Local configurations

$\pi=\alpha_{1}+k \delta \geq \alpha_{1}+2 \delta>\alpha_{1}+\frac{\pi}{2}>\pi$, which is an impossibility. And so $\delta=\frac{\pi}{5}$ and also $k=2$, as indicated in Figure 15. We also have $\alpha_{1}=\frac{3 \pi}{5}$. We shall denote such f-tiling by $\mathcal{N}$. Its 3D representation is shown in Figure 16.

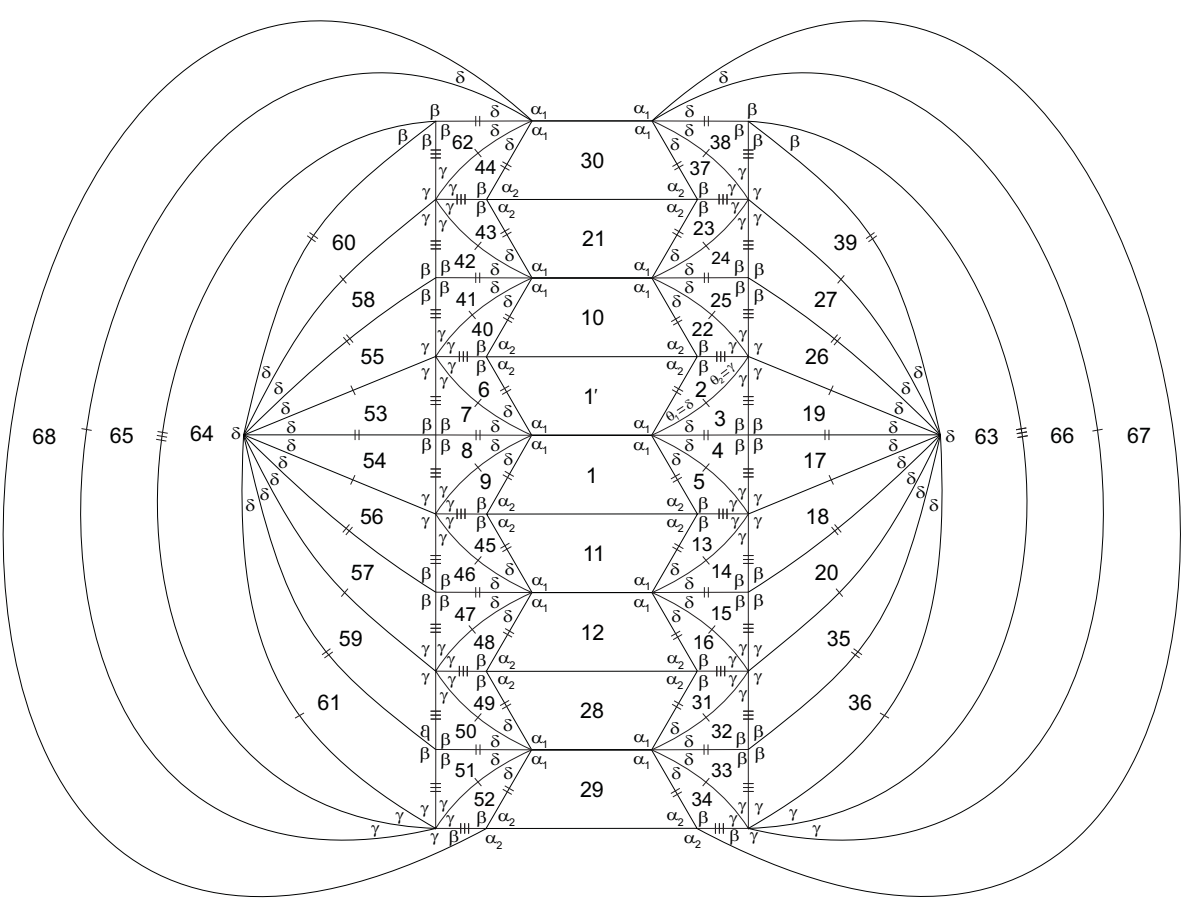

Figure 15: Planar representation of $\mathcal{N}$

2. If $\theta_{2}=\beta$ (Figure $8(\mathrm{a})$ ), then we obtain the local configuration illustrated in Figure 17(a). Note that $v_{1}$ must be enclosed exclusively by angles $\gamma$ and $\delta$. Now, we have

$$
\theta_{3}=\gamma, \quad \theta_{3}=\alpha_{2} \quad \text { or } \quad \theta_{3}=\beta .
$$

2.1 Suppose firstly that $\theta_{3}=\gamma$. Then $\beta+\gamma=\pi$. Now, if there is at least one angle $\gamma$ 


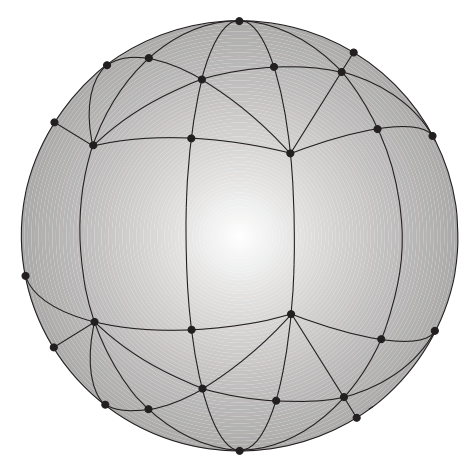

Figure 16: 3D representation of $\mathcal{N}$

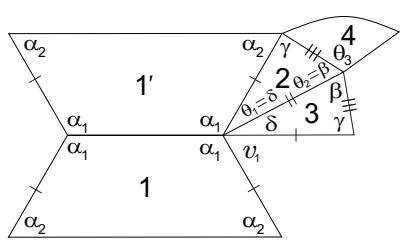

(a)

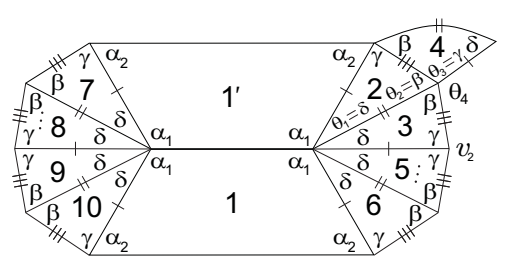

(b)

Figure 17: Local configurations

in the alternating angle sum containing $\alpha_{1}$ (see vertex $v_{1}$, Figure $17(\mathrm{a})$ ), we get $\beta>\alpha_{1}$, and so $\beta+\alpha_{2}>\pi$, which is not possible (see tile 4). Therefore $\alpha_{1}+k \delta=\pi$, for some $k \geq 2$, and we get the local configuration illustrated in Figure 17(b). We also obtain the following relation between angles:

$$
\alpha_{1}>\beta>\frac{\pi}{2}>\gamma \geq \alpha_{2}>\delta
$$

Now, $\theta_{4}$ must be $\alpha_{2}$ or $\gamma$.

2.1.1 If $\theta_{4}=\alpha_{2}$ (and consequently $\gamma=\alpha_{2}$ ) and the vertex $v_{2}$ has valency six, we get the local configuration illustrated in Figure 18(a) and consequently a contradiction at vertex $v_{3}$. If vertex $v_{2}$ has valency eight, we reach a contradiction as we can observe at Figure 18(b) (vertex $v_{4}$ ). Using the same kind of reasoning when $v_{2}$ has valency greater than eight, we also obtain a contradiction.

2.1.2 If $\theta_{4}=\gamma$, we obtain the configuration illustrated in Figure 19(a) and now we have $\theta_{5}=\alpha_{2}$ or $\theta_{5}=\gamma$.

2.1.2.1 If $\theta_{5}=\alpha_{2}$ (therefore $\alpha_{2}=\gamma$ ), the last configuration is extended in a unique way to the one illustrated in Figure 19(b). In this configuration we have obtained two vertices surrounded by the cyclic sequence of angles $\left(\alpha_{2}, \gamma, \alpha_{2}, \gamma, \alpha_{2}, \gamma, \ldots\right)$, with $\alpha_{2}=\gamma=\frac{\pi}{k}$, for some $k \geq 3$. We have considered $k=3$ for convenience. Although a complete planar representation was possible to draw, we may conclude that such a configuration cannot be realized by an f-tiling since there is no spherical trapezoid satisfying the relations that come from Figure 19(b). In fact, we have $Q=T \cup T^{\prime}$ (Figure 20) and then $\beta+x=\alpha_{2}$, 


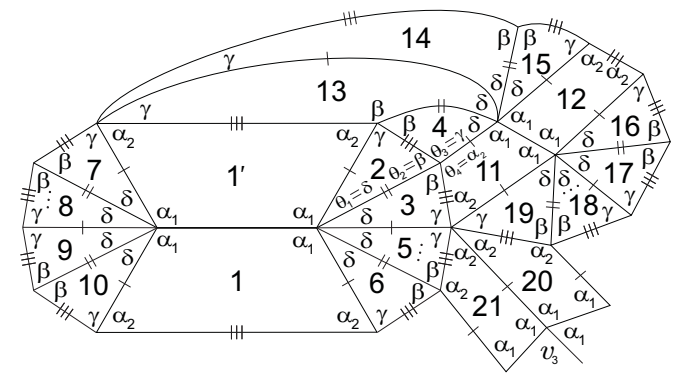

(a)

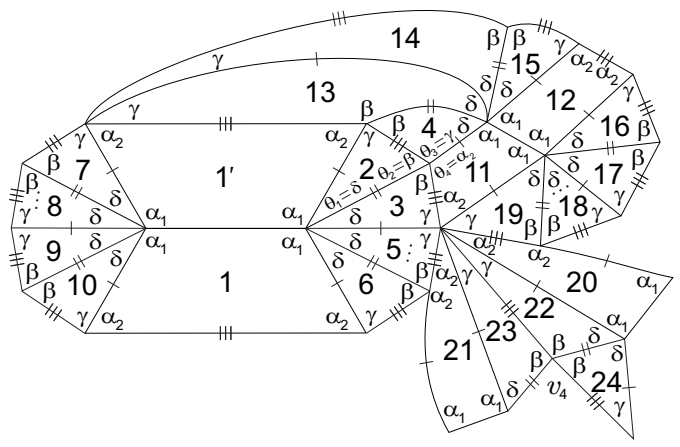

(b)

Figure 18: Local configurations

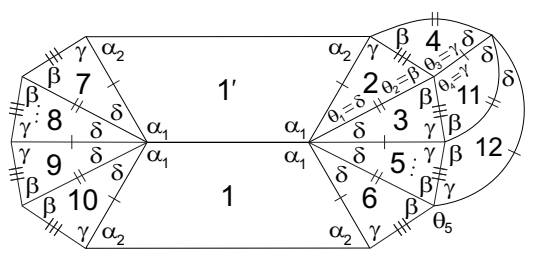

(a)

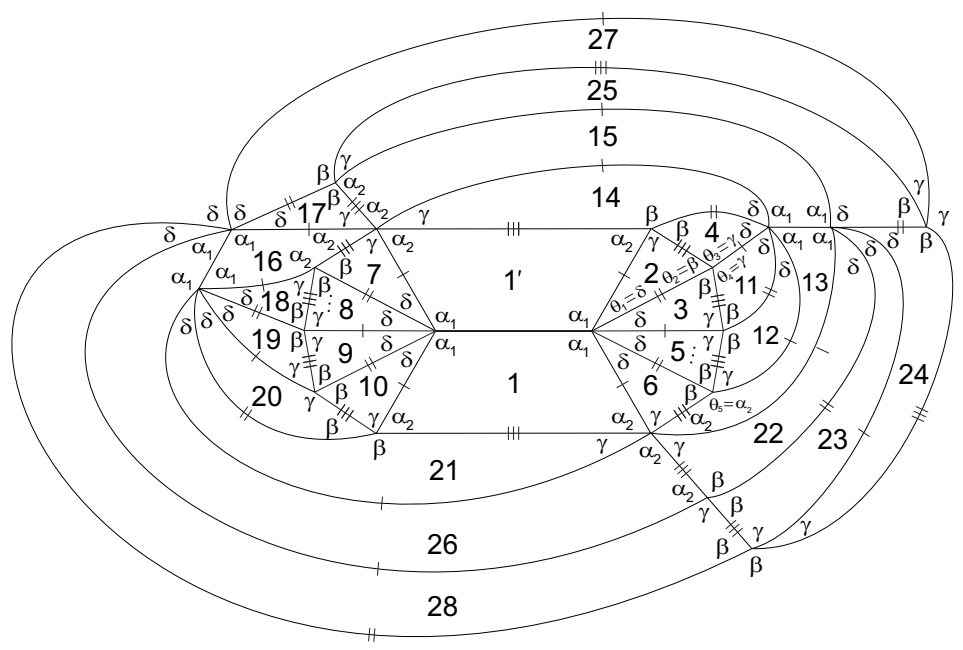

(b)

Figure 19: Local configurations

for some $x>0$, which is a contradiction, as $\beta>\alpha_{2}$.

2.1.2.2 If $\theta_{5}=\gamma$, then the last configuration is extended to the one illustrated in Figure 21(a). Using similar arguments to the ones used before in 2.1.2.1, we conclude that the case $\theta_{6}=\alpha_{2}$ (Figure 21(b)) leads to a contradiction (by using an argument of symmetry). On the other hand, the case $\theta_{6}=\gamma$ leads the the configuration illustrated in Figure 22(a) (by using arguments of symmetry). Now, if tile 18 is a triangle, then it must be set up 

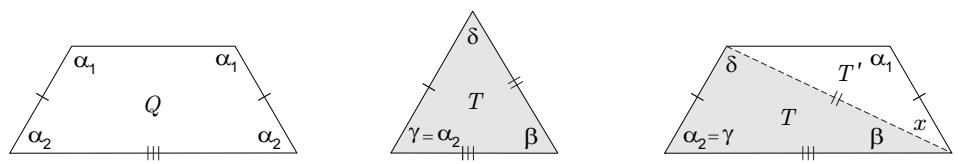

Figure 20: $Q=T \cup T^{\prime}$

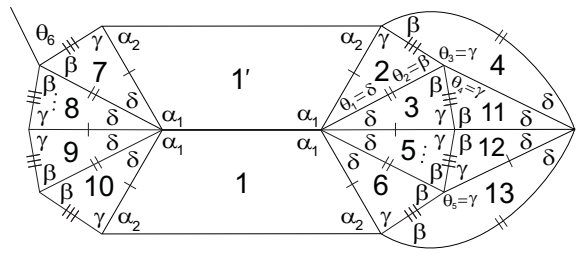

(a)

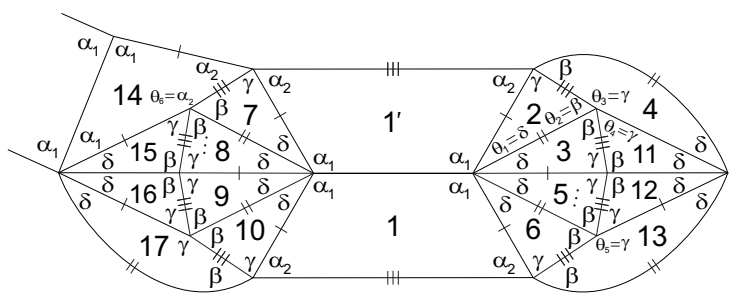

(b)

Figure 21: Local configurations

as indicated in Figure 22(b); however there is no way to satisfy the angle-folding relation around vertex $v$ (see length sides). If tile 18 is a trapezoid (Figure 23(a)), we also reach

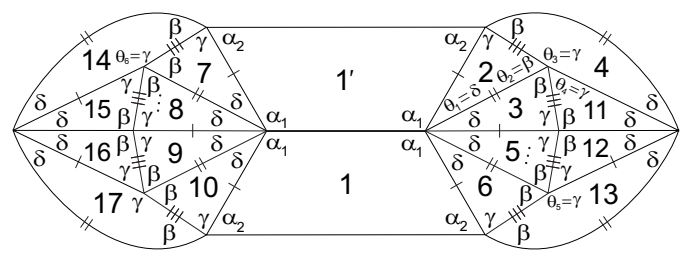

(a)

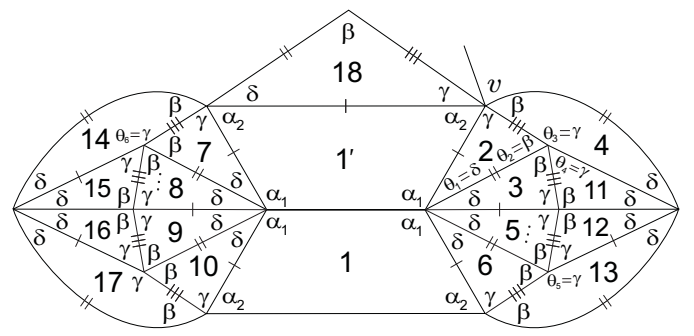

(b)

Figure 22: Local configurations

a contradiction at vertex $v$, as $\gamma+\alpha_{2}+\beta>\pi$.

2.2 Consider now that $\theta_{3}=\alpha_{2}$ (Figure 23(b)). In this case we may conclude that $\alpha_{1}>$ $\beta \geq \frac{\pi}{2}>\gamma \geq \alpha_{2}>\delta$.

If $\beta+\alpha_{2}<\pi$ (Figure 24(a)), then $\gamma>\alpha_{2}$ and also $\alpha_{1}+k \delta=\pi$, for some $k \geq 2$. At vertex $v$ we obtain $\beta+\alpha_{2}<\pi$ and $\beta+\gamma=\pi$, which is an impossibility.

On the other hand, if $\beta+\alpha_{2}=\pi$, we get the configuration illustrated in Figure 24(b), 


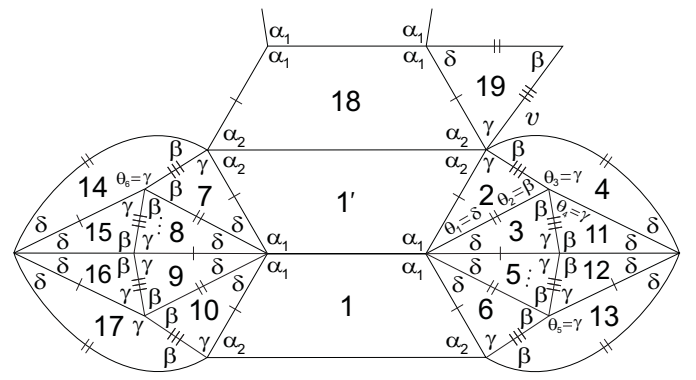

(a)

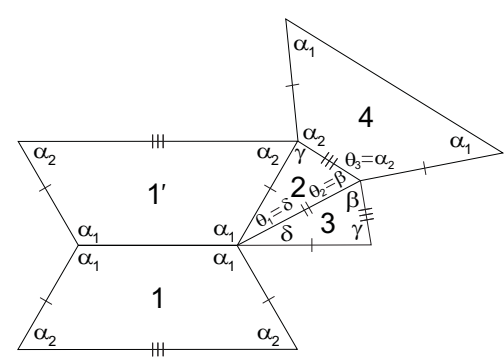

(b)

Figure 23: Local configurations

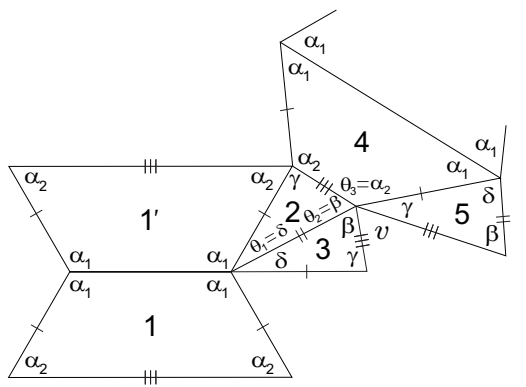

(a)

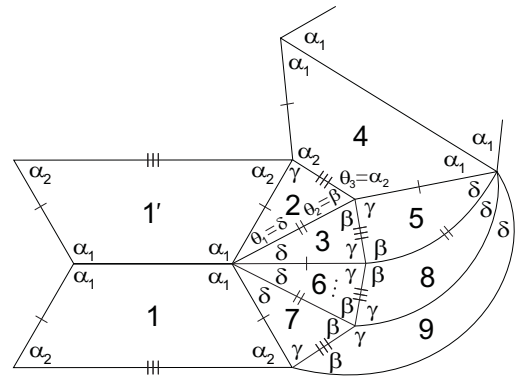

(b)

Figure 24: Local configurations

with $\alpha_{2}=\gamma$, and $\alpha_{1}+k \delta=\pi$, for some $k \geq 2$. As in 2.1.2.1, we obtain a contradiction. 2.3 Finally, we consider the case $\theta_{3}=\beta$. Now, we get the configuration illustrated in Figure 25(a). It results that $\beta=\frac{\pi}{2}\left(\gamma+\delta>\frac{\pi}{2}\right)$ which implies that $\alpha_{1}>\beta$ and $\alpha_{1}+k \delta=\pi$, for some $k \geq 2$. Now, at vertex $v$ we must have $2 \gamma+\alpha_{2}=\pi$ or $2 \gamma+\delta=\pi$

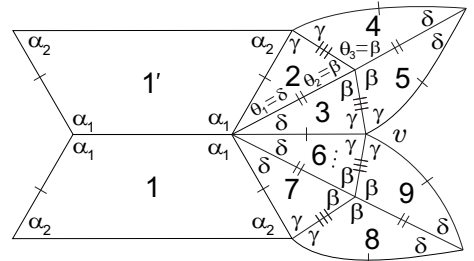

(a)

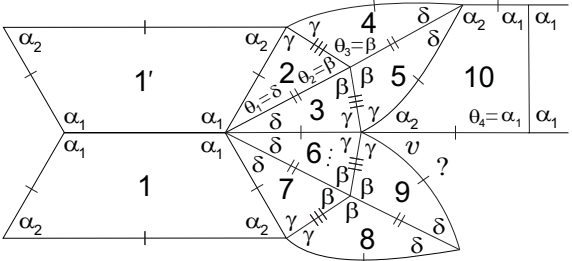

(b)

Figure 25: Local configurations

or $3 \gamma=\pi$.

2.3.1 Consider firstly that $2 \gamma+\alpha_{2}=\pi$. Accordingly to the edge lengths, if $\theta_{4}=\alpha_{1}$ (Figure 25(b)), the sums of alternating angles at vertex $v$ cannot be defined. Therefore, $\theta_{4}=\alpha_{2}$. On the other hand, if $\theta_{5}=\gamma$ (Figure 26(a)), we have $\lambda=\gamma$ or $\lambda=\delta$. In both cases we reach a contradiction, as we can observe in Figure 26(b) and Figure 27(a), 


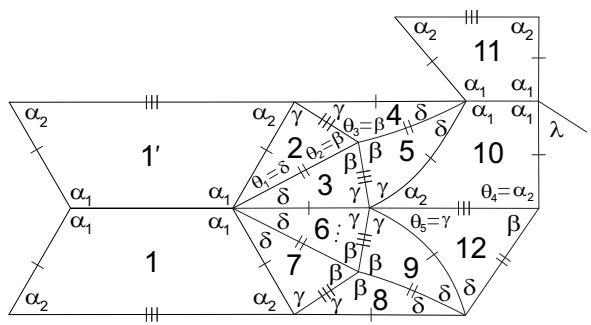

(a)

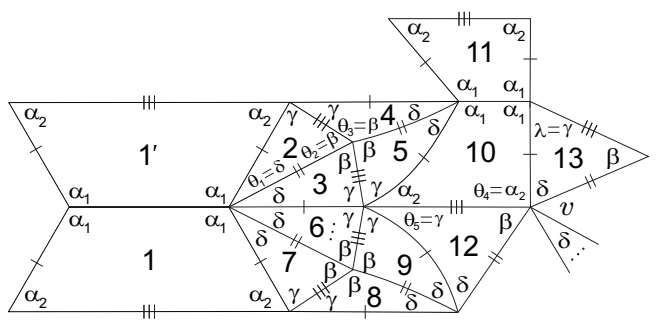

(b)

Figure 26: Local configurations

respectively (in the first case we must have $\beta+k \delta=\pi, k \geq 2$, and so we have no way to avoid an incompatibility between sides at vertex $v$; in the second case we have $\pi=\beta+\gamma<\beta+\beta=\pi$, which is a contradiction). Therefore $\theta_{5}=\alpha_{2}$, and the last

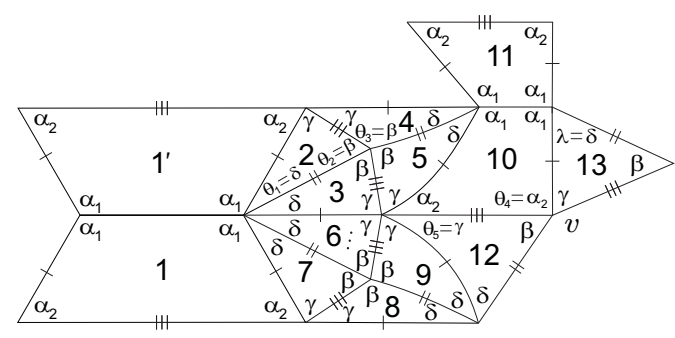

(a)

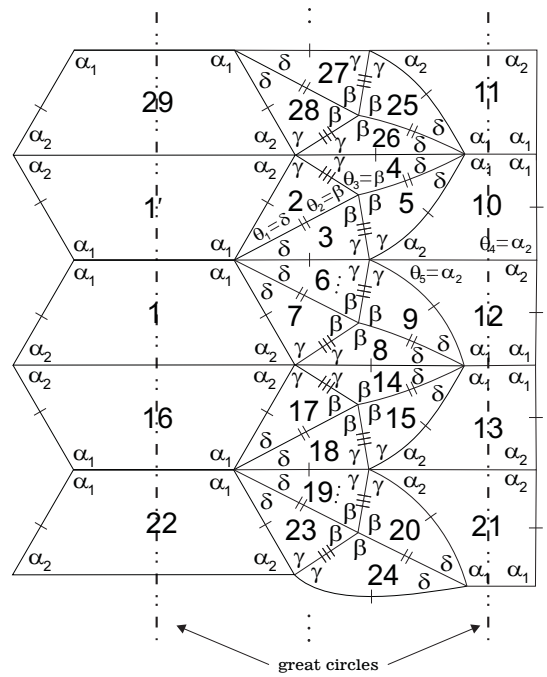

(b)

Figure 27: Local configurations

configuration is extended in a unique way to the one illustrated in Figure 27(b). But then we obtain two great circles in the sphere that do not intersect, which is not possible. 2.3.2 If $2 \gamma+\delta=\pi$, we get the configuration illustrated in Figure 28(a). Suppose that, at vertex $v$, we have $\alpha_{1}+\gamma=\pi$. Then,

$$
\left\{\begin{array} { r l } 
{ \alpha _ { 1 } + k \delta } & { = \pi } \\
{ 2 \gamma + \delta } & { = \pi } \\
{ \alpha _ { 1 } + \gamma } & { = \pi }
\end{array} \quad \Longrightarrow \quad \left\{\begin{array}{rl}
\alpha_{1} & =\frac{(k+1) \pi}{2 k+1} \\
\gamma & =\frac{k \pi}{2 k+1},
\end{array} \quad k \geq 2\right.\right.
$$


The relation between angles is given by

$$
\left\{\begin{array}{c}
\alpha_{1}>\beta=\frac{\pi}{2}>\gamma>\delta \\
\alpha_{1}>\alpha_{2}>\gamma>\delta
\end{array}\right.
$$

Considering a vertex surrounded by the cyclic sequence $\left(\alpha_{2}, \gamma, \gamma, \ldots\right)$, we obtain $\alpha_{2}+\gamma<\pi$ and $\alpha_{2}+\gamma+\rho \geq \alpha_{2}+\gamma+\delta>\frac{2 k \pi}{2 k+1}+\frac{\pi}{2 k+1}=\pi, \forall \rho \in\left\{\alpha_{1}, \alpha_{2}, \beta, \gamma, \delta\right\}$, which is a contradiction. Therefore, at vertex $v$, we have $\alpha_{1}+k \delta=\pi, k \geq 2$.

Now, tile 20 must be a triangle or a trapezoid, but, as illustrated in Figure 28(b) and Figure 29(a), respectively, we reach a contradiction at vertex $v$.

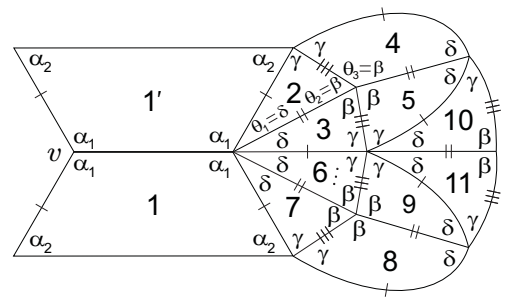

(a)

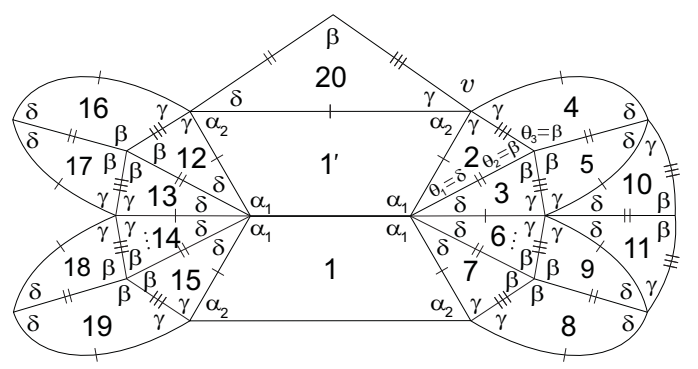

(b)

Figure 28: Local configurations

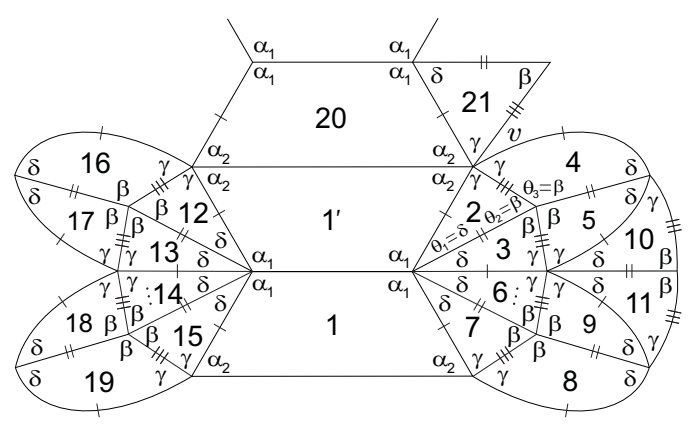

(a)

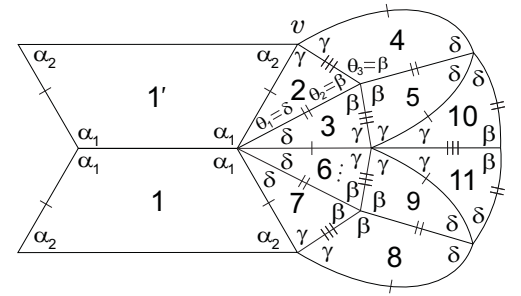

(b)

Figure 29: Local configurations

2.3.3 Finally, if $3 \gamma=\pi$, we obtain the configuration illustrated in Figure 29(b). If we have, at vertex $v, \alpha_{2}+\gamma=\pi$, then $\alpha_{2}=\frac{2 \pi}{3}$. On the other hand, we have $\beta=\frac{\pi}{2}, \gamma=\frac{\pi}{3}$ and $\beta+\gamma+\delta>\pi$. Therefore $\delta>\frac{\pi}{6}$. As $\alpha_{1}+k \delta=\pi$, for some $k \geq 2$, we obtain $\alpha_{1}<\frac{2 \pi}{3}$, which is a contradiction $\left(\alpha_{1}>\alpha_{2}\right)$. Thus, we must have $\alpha_{2}+\gamma<\pi$ and the last configuration is extended to the one illustrated in Figure 30(a). Note that, at vertex $v_{1}$, we cannot have $\alpha_{1}+\gamma=\pi$, as this condition implies $\gamma=\frac{\pi}{3}=2 \delta$, and so $\beta+\gamma+\delta=\frac{\pi}{2}+\frac{\pi}{3}+\frac{\pi}{6}=\pi$, which is a contradiction. 


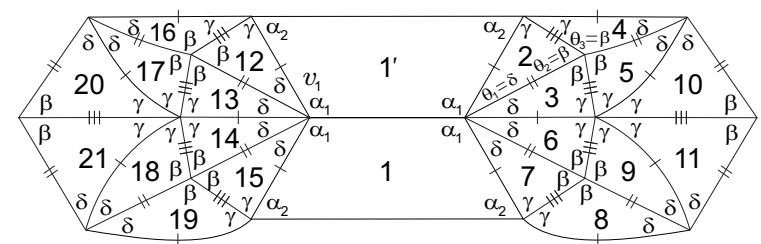

(a)

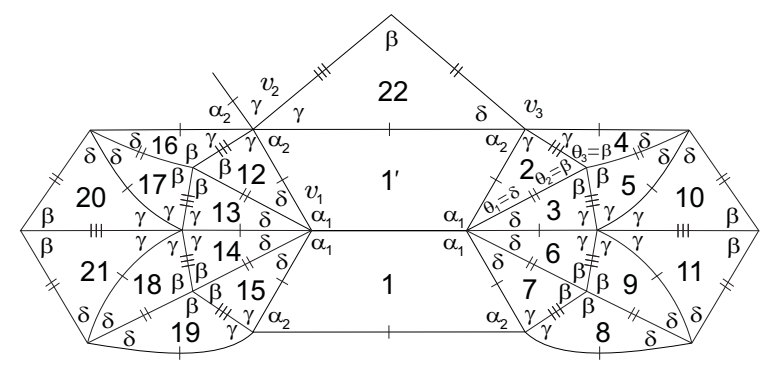

(b)

Figure 30: Local configurations

Now, if tile 22 is a triangle (Figure $30(\mathrm{~b})$ ), then $v_{2}$ and $v_{3}$ have valency six, with $\alpha_{2}=\gamma$. Nevertheless, an incompatibility between angles takes place at vertex $v_{3}$. Thus, tile 22 is a trapezoid and, using similar argumentation, the last configuration is extended in a unique way to the one illustrated in Figure 31. We obtain $k=2, \beta=\frac{\pi}{2}, \alpha_{2}=\gamma=\frac{\pi}{3}$,

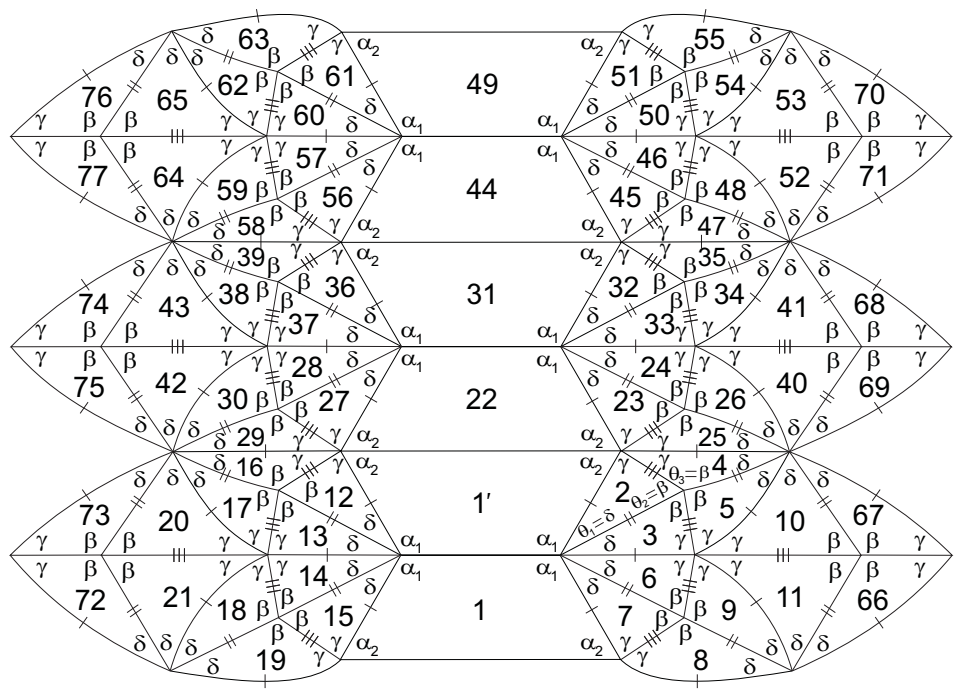

Figure 31: Local configuration

$\alpha_{1}=\frac{3 \pi}{5}$ and $\delta=\frac{\pi}{5}$. But then $\alpha_{1}+\alpha_{2}=\frac{3 \pi}{5}+\frac{\pi}{3}=\frac{14 \pi}{15}<\pi$, which is a contradiction.

Proposition 2.3 If $\theta_{1}=\delta$ and $\alpha_{1}+\delta=\pi$ (Figure 3), then $\Omega(Q, T) \neq \emptyset$ iff

(i) $\alpha_{2}+\gamma=\pi$ and $\beta=\frac{\pi}{2} \quad$ or

(ii) $\alpha_{2}+2 \gamma=\pi, \beta=\frac{\pi}{2}$ and $k \delta=\pi$, for some $k \geq 4$, or

(iii) $\alpha_{2}+\beta=\pi$ and $k \gamma=\pi$, for some $k \geq 3$. 
The first case leads to a family of f-tilings, denoted by $\mathcal{R}_{\delta \gamma}^{2}$, with $\delta, \gamma \in\left(0, \frac{\pi}{2}\right)$. A planar representation is given in Figure 38(a). For its 3D representation see Figure 38(b).

The case (ii) leads to a family of f-tilings, denoted by $\mathcal{M}_{\gamma}^{k}$, with

$$
\gamma \in\left(\frac{(k-2) \pi}{2 k}, \frac{1}{2} \arccos \left(-\cos ^{2} \frac{\pi}{k}\right)\right) .
$$

In Figure 41 is given the corresponding planar representation. 3D representations for $k=4$ and $k=5$ are given in Figure 42.

In the last situation, there is a family of f-tilings, denoted by $\mathcal{R}_{\delta \beta}^{k}$, with

$\beta+\delta \in\left(\frac{(k-1) \pi}{k}, \frac{\pi}{k}+\arccos \left(-\cos ^{2} \frac{\pi}{k}\right)\right)$ and $k \geq 3$. A planar representation is given in Figure 52. For their $k=3, k=4$ and $k=53 D$ representations see Figure 53.

Proof. Suppose that we have two cells in adjacent positions as illustrated in Figure 3 and consider $\theta_{1}=\delta$, with $\alpha_{1}+\delta=\pi$. With the labelling of Figure 32(a), we have

$$
\theta_{2}=\beta \quad \text { or } \quad \theta_{2}=\gamma
$$

1. Suppose firstly that $\theta_{2}=\beta$ (Figure $32(\mathrm{~b})$ ). Now, we have

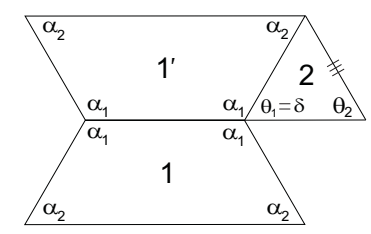

(a)

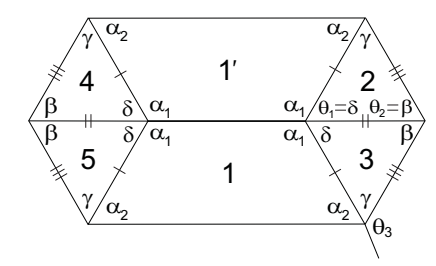

(b)

Figure 32: Local configurations

$$
\theta_{3}=\alpha_{2}, \quad \theta_{3}=\beta \quad \text { or } \quad \theta_{3}=\gamma
$$

1.1 If $\theta_{3}=\alpha_{2}$, we have necessarily $\alpha_{2}+\beta=\pi$ and $\alpha_{1}>\beta>\alpha_{2}=\gamma>\delta$, and the last local configuration is extended in a unique way to the one illustrated in Figure 33.

One gets three distinct types of vertices and the sums of alternated angles around them are, respectively,

$$
\alpha_{1}+\delta=\pi, \quad \alpha_{2}+\beta=\pi=\beta+\gamma \quad \text { and } \quad k \alpha_{2}=\pi=k \gamma
$$

for some $k \geq 3$. Consequently,

$$
\alpha_{1}=\pi-\delta, \quad \alpha_{2}=\gamma=\frac{\pi}{k} \quad \text { and } \quad \beta=\frac{(k-1) \pi}{k} .
$$




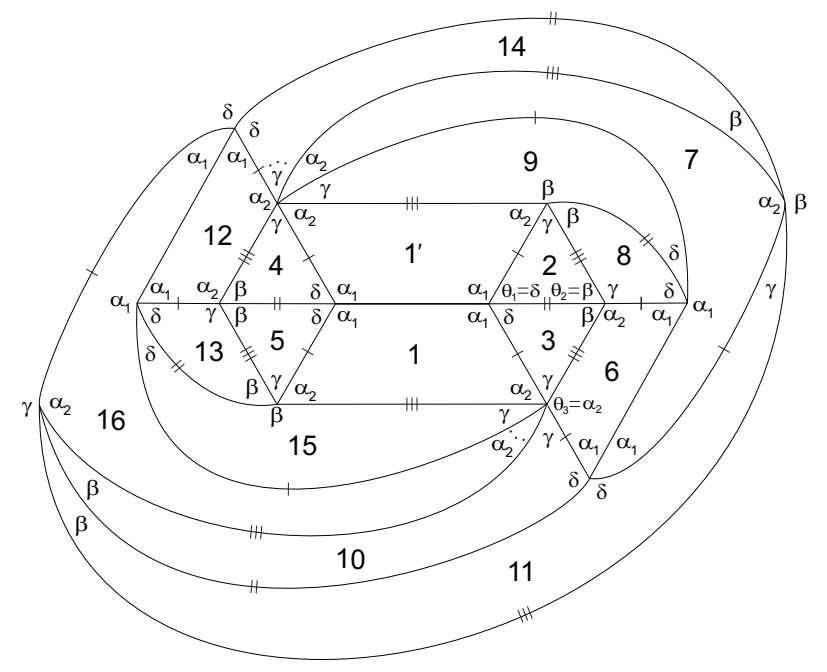

Figure 33: Local configuration
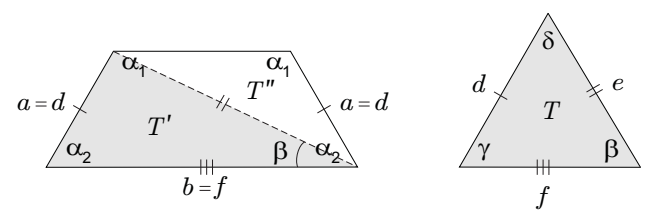

Figure 34: $Q=T^{\prime} \cup T^{\prime \prime}$

In Figure 34, the spherical trapezoid is divided in two triangles, $T^{\prime}$ and $T^{\prime \prime}$. Since $T$ and $T^{\prime}$ have two sides in common and the angle formed by these sides is equal $\left(\alpha_{2}=\gamma\right)$, we conclude that $T$ and $T^{\prime}$ are congruent. Now, as $Q=T^{\prime} \cup T^{\prime \prime}$, we have $\alpha_{2}>\beta$, which is an incongruity. Thus, we conclude that the extended planar configuration given in Figure 33 does not correspond to any f-tiling.

1.2 If $\theta_{3}=\beta$ (Figure 35(a)), we consider separately the cases $\alpha_{2}+\beta=\pi$ and $\alpha_{2}+\beta<\pi$.

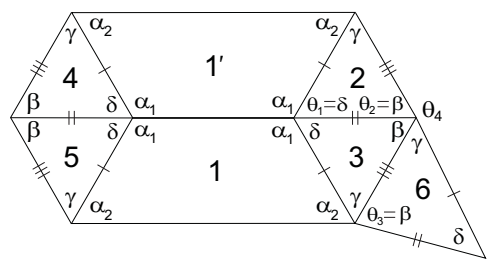

(a)

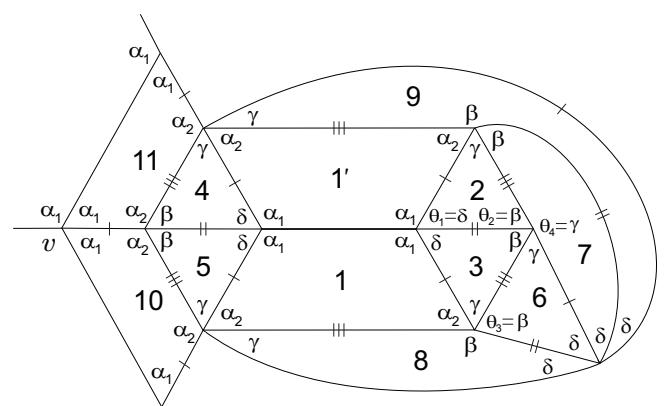

(b)

Figure 35: Local configurations

(i) If $\alpha_{2}+\beta=\pi$, then $\alpha_{1}>\beta>\alpha_{2}=\gamma>\delta$. Now, if $\theta_{4}=\alpha_{2}$, we end up to a contradiction 
since we obtain the planar representation given in Figure 33 (in a symmetrical way). On the other hand, if $\theta_{4}=\gamma$, we reach an impossibility at vertex $v$, see Figure 35(b).

(ii) If $\alpha_{2}+\beta<\pi$, then $\alpha_{1}>\beta>\gamma>\alpha_{2}>\delta$, and

$$
\theta_{4}=\alpha_{2}, \quad \theta_{4}=\gamma \quad \text { or } \quad \theta_{4}=\delta \quad(\text { Figure } 36(\mathrm{a}))
$$

In all these cases we obtain a contradiction, as we can observe in Figures 36(b), 37(a) and 37 (b), where we have no way to avoid an incompatibility at vertex $v$.

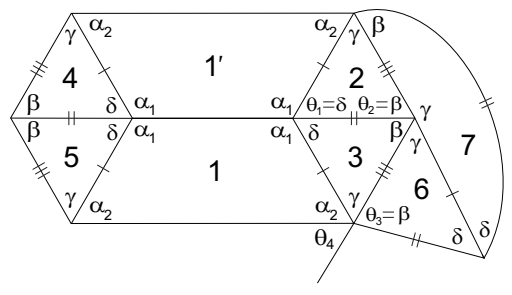

(a)

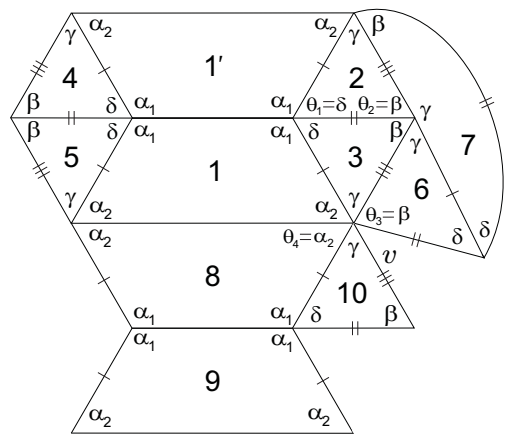

(b)

Figure 36: Local configurations

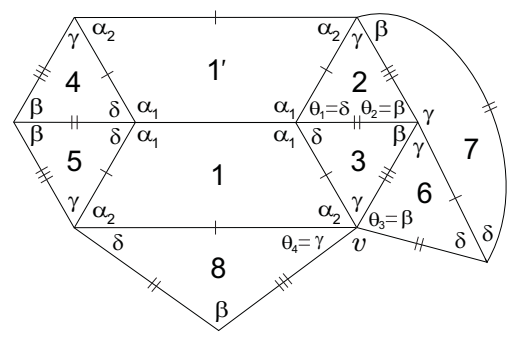

(a)

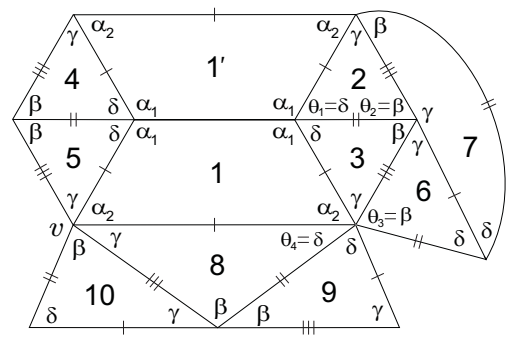

(b)

Figure 37: Local configurations

1.3 If $\theta_{3}=\gamma$ (Figure 32(b)), then $\beta+\beta=\pi$, i.e., $\beta=\frac{\pi}{2}$, and hence $\gamma+\delta>\frac{\pi}{2}$. Now, we consider separately the cases $\alpha_{2}+\gamma=\pi$ and $\alpha_{2}+\gamma<\pi$.

(i) If $\alpha_{2}+\gamma=\pi$, then $\alpha_{1}>\alpha_{2}>\beta=\frac{\pi}{2}>\gamma>\delta$ and the refereed local configuration is extended in a unique way to the one given in Figure 38(a), where $\delta, \gamma \in\left(0, \frac{\pi}{2}\right)$. The corresponding 3D representation is illustrated in Figure 38(b). We denote such family of f-tilings by $\mathcal{R}_{\delta \gamma}^{2}$, with $\delta, \gamma \in\left(0, \frac{\pi}{2}\right), \delta<\gamma$.

(ii) If $\alpha_{2}+\gamma<\pi$, then, with the labelling of Figure 39(a), we have

$$
\theta_{4}=\gamma, \quad \theta_{4}=\alpha_{2} \quad \text { or } \quad \theta_{4}=\delta
$$




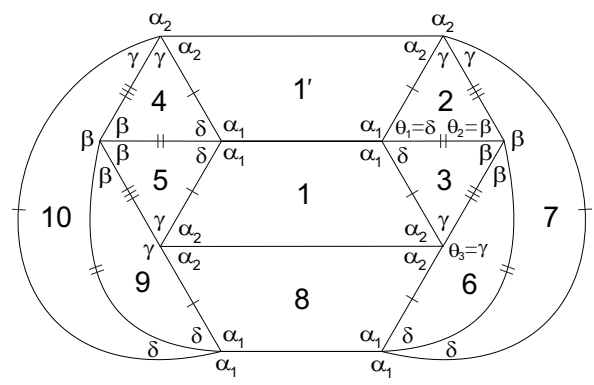

(a) Planar representation

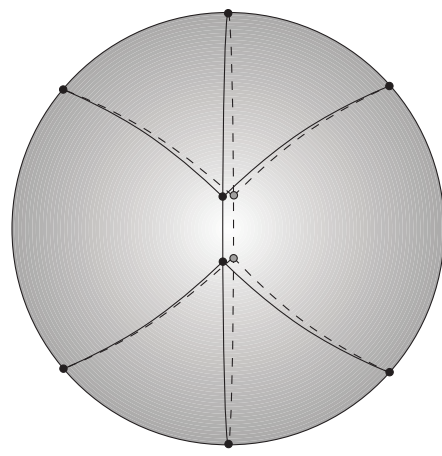

(b) 3D representation

Figure 38: f-tilings $\mathcal{R}_{\delta \gamma}^{2}, \delta, \gamma \in\left(0, \frac{\pi}{2}\right)$

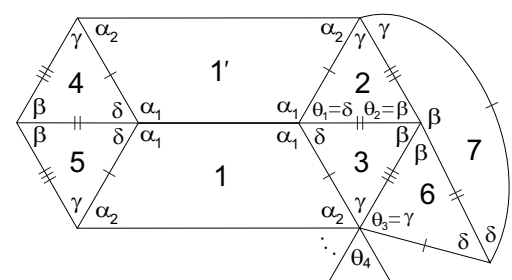

(a)

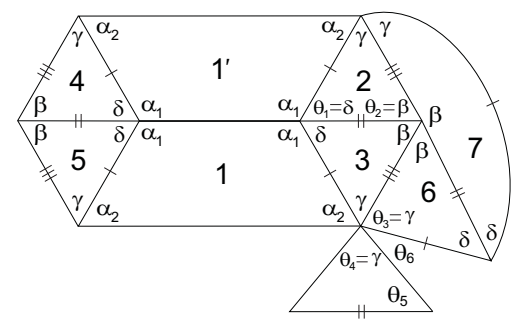

(b)

Figure 39: Local configurations

a) If $\theta_{4}=\gamma$ (Figure 39(b)), we have $\theta_{5}=\beta$ or $\theta_{5}=\delta$.

If $\theta_{5}=\beta$ and considering $\theta_{6}=\alpha_{2}$, we obtain one of the configurations illustrated in Figure 40 (depending on tile 10). In both cases we reach a contradiction at vertex $v$. Considering now $\theta_{6}=\gamma$, the last configuration is extended in a unique way to the one

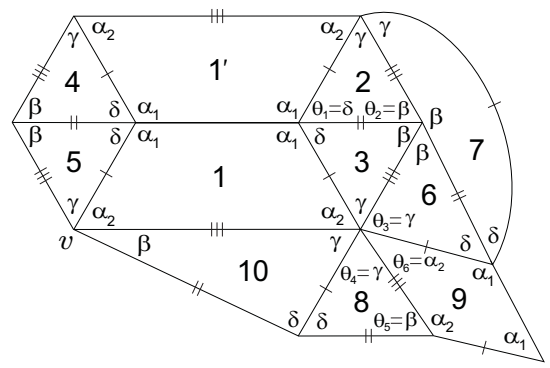

(a)

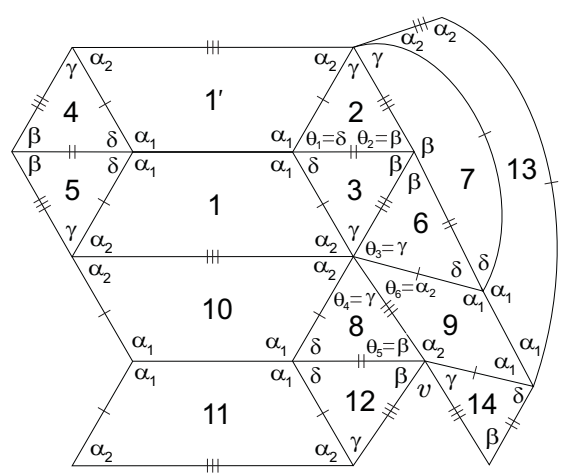

(b)

Figure 40: Local configurations 
illustrated in Figure 41, where the sums of alternated angles around vertices satisfy

$$
\alpha_{1}+\delta=\pi, \quad \beta=\frac{\pi}{2}, \quad \alpha_{2}+2 \gamma=\pi \quad \text { and } \quad k \delta=\pi,
$$

for some $k \geq 4$. The $\mathrm{f}$-tiling with such a planar representation will be denoted by $\mathcal{M}_{\gamma}^{k}, k \geq$

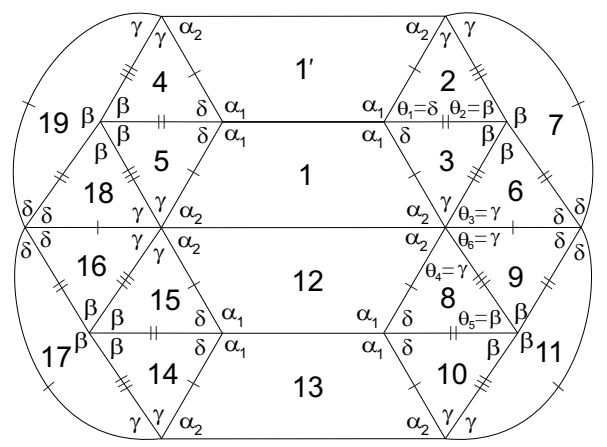

Figure 41: Planar representation of $\mathcal{M}_{\gamma}^{k}$

4. Using spherical trigonometry formulas and by an area $\operatorname{argument}\left(\cos 2 e=\frac{\cos 2 \gamma+\cos ^{2} \delta}{\sin ^{2} \delta}>\right.$ 0 and $A(T)>0$ ), we may conclude that

$$
\gamma \in\left(\frac{(k-2) \pi}{2 k}, \frac{1}{2} \arccos \left(-\cos ^{2} \frac{\pi}{k}\right)\right) .
$$

3D representations of $\mathcal{M}_{\gamma}^{4}$ and $\mathcal{M}_{\gamma}^{5}$ are given in Figure 42.

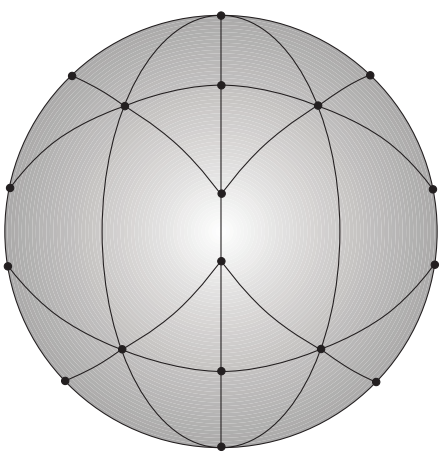

(a) $k=4$

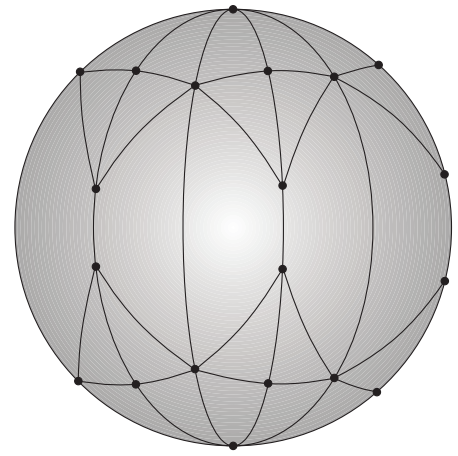

(b) $k=5$

Figure 42: 3D representations of $\mathcal{M}_{\gamma}^{k}, \gamma \in\left(\frac{(k-2) \pi}{2 k}, \frac{1}{2} \arccos \left(-\cos ^{2} \frac{\pi}{k}\right)\right), k \geq 4$

If $\theta_{5}=\delta$ and considering $\theta_{6}=\alpha_{2}$ we reach a contradiction at vertex $v$, see Figure 43(a) (note that we have only considered the case $\theta_{7}=\delta$, as $\theta_{7}=\alpha_{2}$ leads immediately to a contradiction). On the other hand, if $\theta_{6}=\alpha_{1}$ we obtain the configuration illustrated in Figure 43(b). At vertex $v$, we must have $\alpha_{2}+\gamma+k \delta=\pi$, for some $k \geq 2$. Nevertheless, 


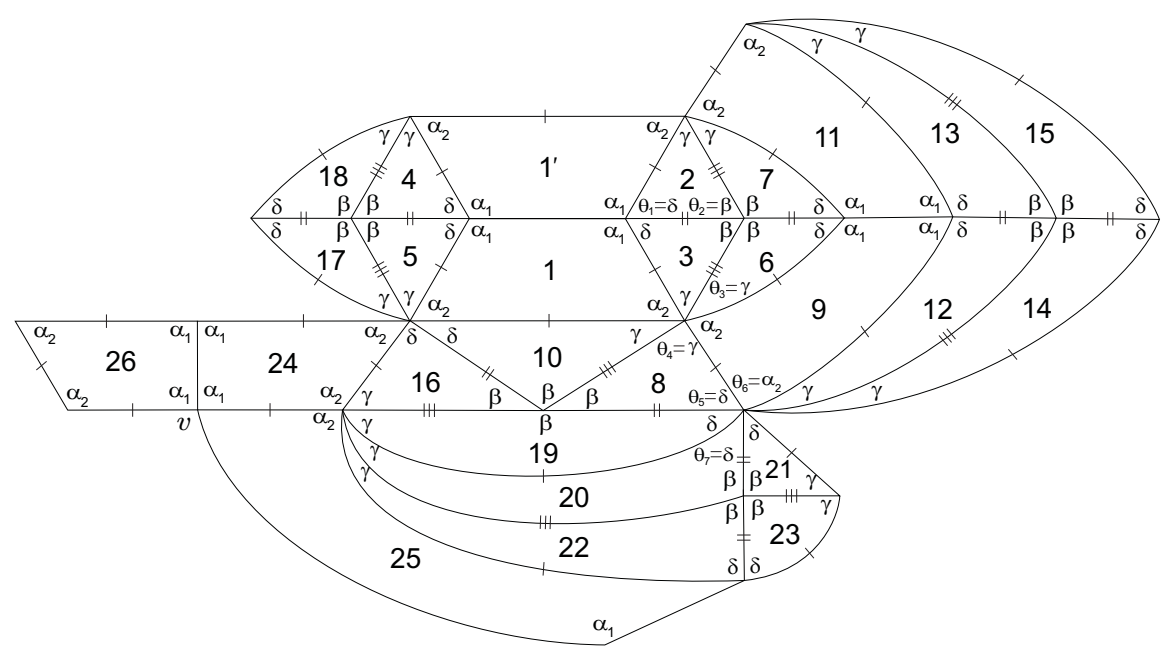

(a)

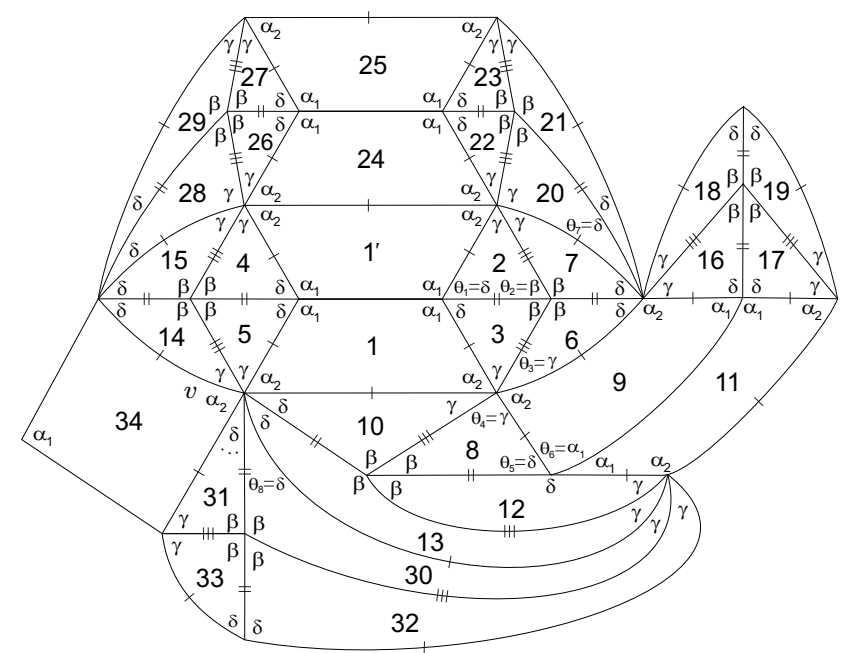

(b)

Figure 43: Local configurations

we obtain a contradiction, as $\alpha_{1}+2 \delta>\pi$ and $\alpha_{1}+\gamma>\pi$ (similarly to the previous case, we must have $\theta_{7}=\theta_{8}=\delta$ ).

b) Consider now that $\theta_{4}=\alpha_{2}$. If $\theta_{5}=\alpha_{1}$ (Figure 44(a)), we reach a contradiction since there is no way to satisfy the angle-folding relation around vertex $v$. On the other hand, if $\theta_{5}=\alpha_{2}$, then the valency of vertex $v_{1}$ must be six (note that $2 \gamma+2 \delta>\pi$ ). Nevertheless, we obtain a contradiction at vertex $v_{2}$, since $\beta+\gamma<\pi=2 \beta$ and $\beta+\gamma+\rho>\pi$, for any $\rho \in\left\{\alpha_{1}, \alpha_{2}, \beta, \gamma, \delta\right\}$.

c) Now, if $\theta_{4}=\delta$ (Figure $45(\mathrm{a})$ ), we have $\theta_{5}=\beta$ or $\theta_{5}=\gamma$.

If $\theta_{5}=\beta$, we must have $\theta_{6}=\delta$ (as we can observe in Figure $45(\mathrm{~b})$, the case $\theta_{6}=\alpha_{2}$ leads to a contradiction at vertex $v$ ). Now, we have $\theta_{7}=\alpha_{2}$ or $\theta_{7}=\beta$ (Figure 46(a)) .

If $\theta_{7}=\alpha_{2}$, we obtain a contradiction as illustrated in Figure 46(b) (see vertex $v$ ). Thus 


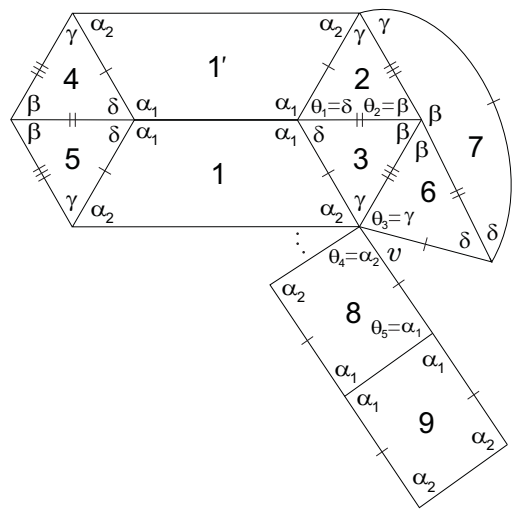

(a)

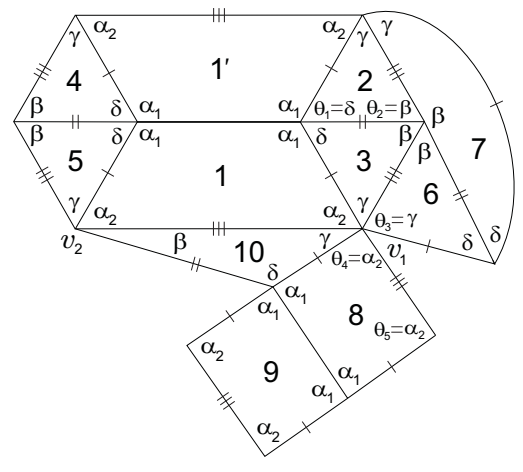

(b)

Figure 44: Local configurations

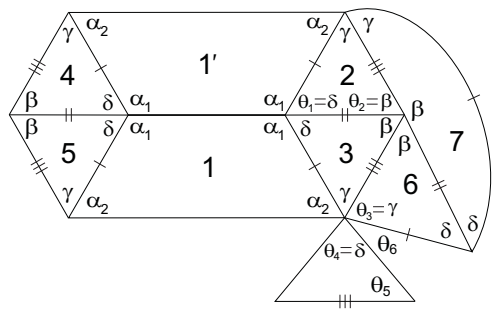

(a)

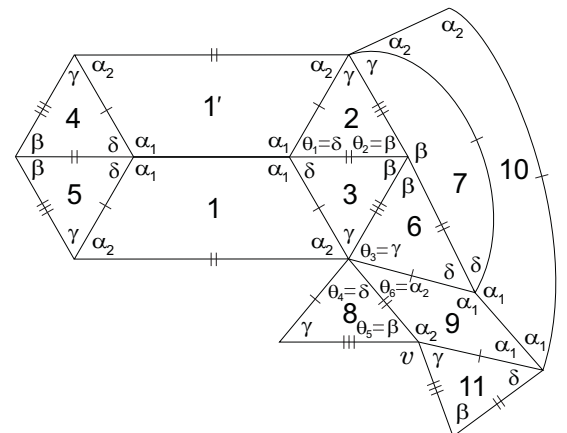

(b)

Figure 45: Local configurations

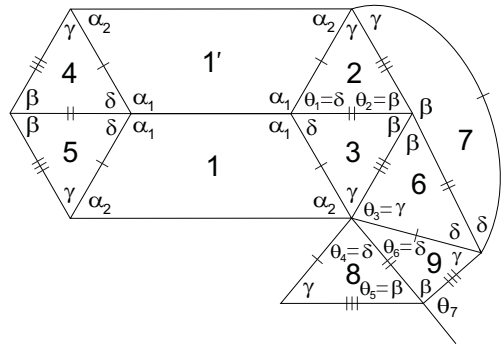

(a)

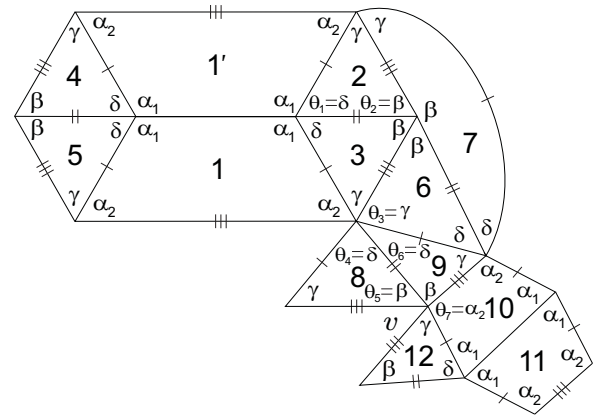

(b)

Figure 46: Local configurations

$\theta_{7}=\beta$ and the last configuration is extended to get the one illustrated in Figure $47(\mathrm{a})$. Note that $v_{1}$ cannot have valency six, since the possible choices for $\theta_{8}$, namely $\delta, \alpha_{2}$ and $\gamma$, lead to contradictions at vertices $v_{2}$ and $v_{3}$. 


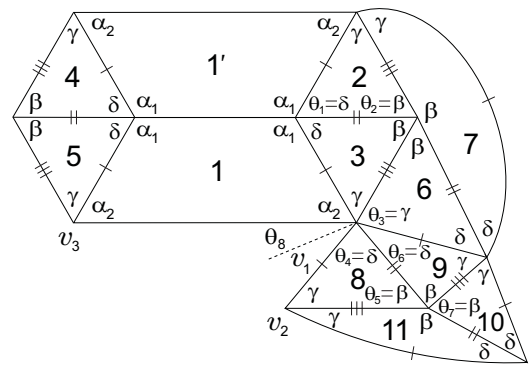

(a)

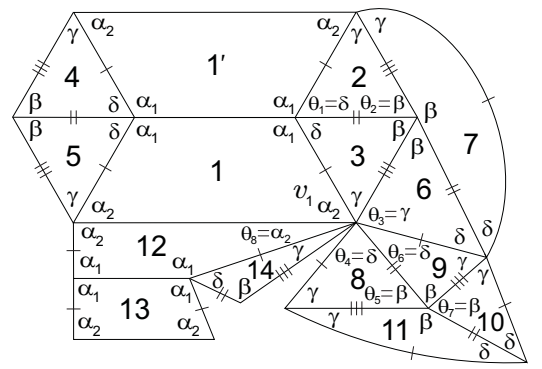

(b)

Figure 47: Local configurations

Now, if $\theta_{8}=\alpha_{2}$, we obtain the configuration illustrated in Figure 47(b). At vertex $v_{1}$, one of the alternating angle sums must contain the sequence $\left(\ldots, \gamma, \alpha_{2}, \gamma, \delta, \ldots\right)$, which is an impossibility, as $\gamma+\delta>\frac{\pi}{2}$. On the other hand, if $\theta_{8}=\delta$, we have $\alpha_{2}+\gamma+k \delta=\pi$, for some $k \geq 2$, and we also reach a contradiction, as illustrated in Figure 48(a) (see vertex $\left.v_{4}\right)$.

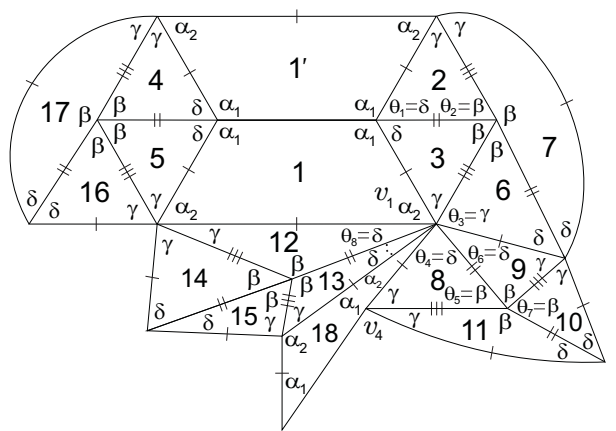

(a)

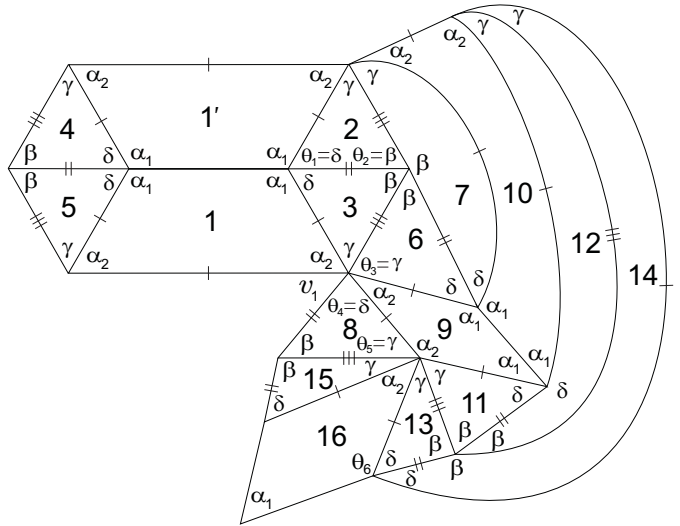

(b)

Figure 48: Local configurations

If $\theta_{5}=\gamma$ (Figure 45(a)), we obtain the configuration illustrated in Figure 48(b). We have necessarily $2 \gamma+\alpha_{2}=\pi$, and consequently $\alpha_{2}<\gamma$ (otherwise at vertex $v_{1}$ we obtain $\alpha_{2}+\gamma+\delta<\pi$ and $\alpha_{2}+\gamma+\delta+\rho>\pi$, for all $\rho$ in $\left\{\alpha_{1}, \alpha_{2}, \beta, \gamma, \delta\right\}$, which is a contradiction).

If $\theta_{6}=\alpha_{1}$ (Figure 48(b)), the last local configuration is extended in a unique way to the one given in Figure 49(a), where, at vertex $v$, we reach a contradiction. On the other hand, if $\theta_{6}=\alpha_{2}$, we obtain the configuration illustrated in Figure 49(b). Again, we reach a contradiction (see vertex $v$ ).

2. Suppose now that $\theta_{2}=\gamma$ (Figure 32(a)). With the labelling of Figure 50(a), we have

$$
\theta_{3}=\alpha_{2}, \quad \theta_{3}=\beta \quad \text { or } \quad \theta_{3}=\gamma .
$$




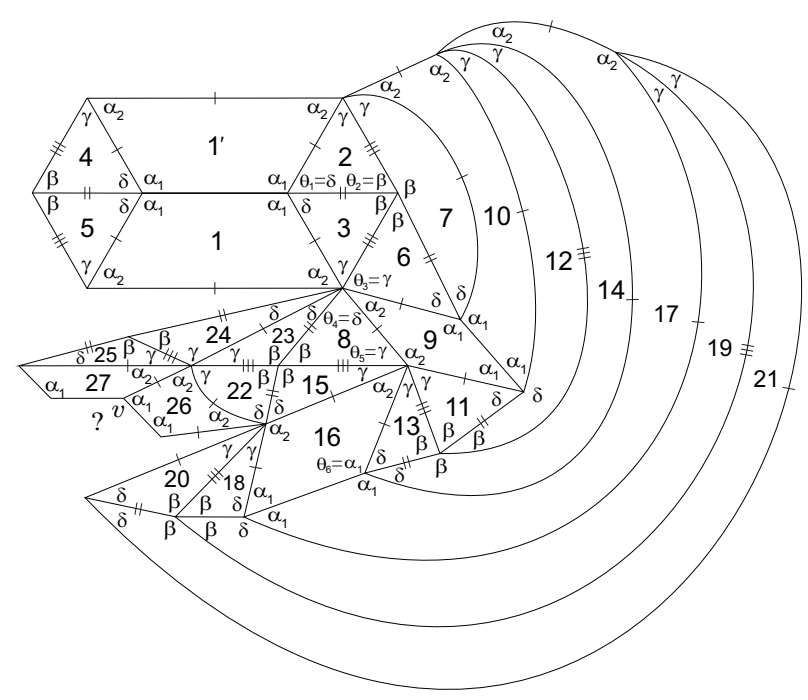

(a)

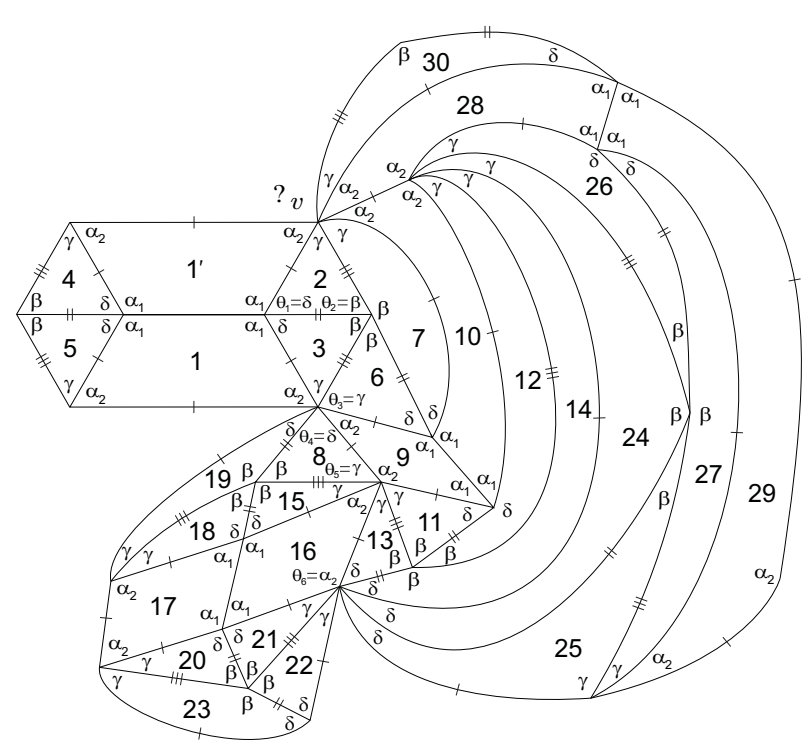

(b)

Figure 49: Local configurations

2.1 If $\theta_{3}=\alpha_{2}$, then $\alpha_{2}=\beta=\frac{\pi}{2}$ and we obtain a contradiction at vertex $v$, see Figure 50(b), as $\beta+\gamma<\pi$ and $\beta+\gamma+\rho>\pi$, for all $\rho$ in $\left.\left\{\alpha_{1}, \alpha_{2}, \beta, \gamma, \delta\right\}\right)$.

2.2 If $\theta_{3}=\beta$ (Figure 50(a)), then we consider separately the cases $\alpha_{2}+\beta=\pi$ and $\alpha_{2}+\beta<\pi$.

(i) If $\alpha_{2}+\beta=\pi$, then the last configuration is extended to get the one illustrated in Figure 51(a).

If $\theta_{4}=\alpha_{2}$, we obtain the local configuration illustrated in Figure 51(b) and consequently we reach a contradiction at vertex $v_{4}$.

If $k \gamma=\pi\left(\theta_{4}=\gamma\right)$, for some $k \geq 3$, then we get the configuration illustrated in 


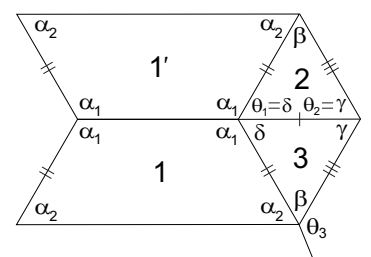

(a)

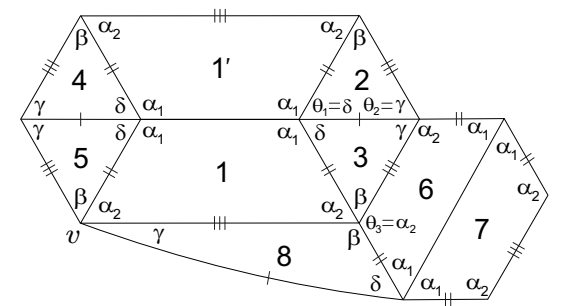

(b)

Figure 50: Local configurations

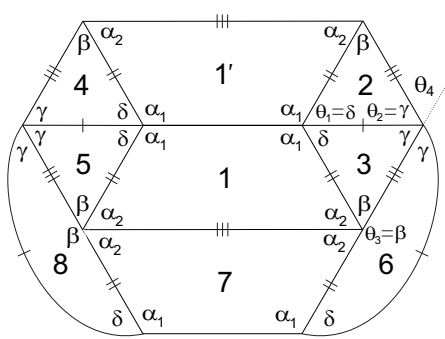

(a)

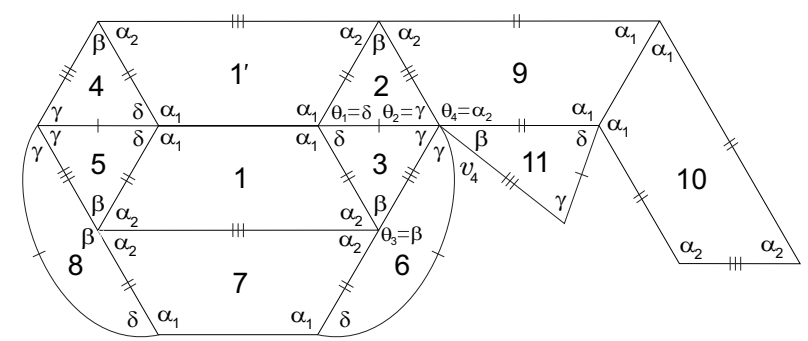

(b)

Figure 51: Local configurations

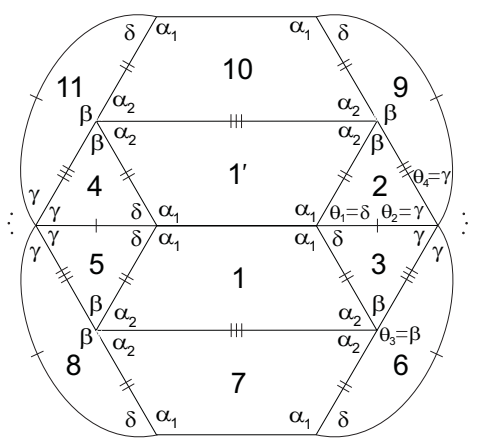

Figure 52: Planar representation of $\mathcal{R}_{\delta \beta}^{k}$

Figure 52. As $\alpha_{1}+\alpha_{2}>\pi$ and by an area argument, we may conclude that $\beta+\delta \in$ $\left(\frac{(k-1) \pi}{k}, \frac{\pi}{k}+\arccos \left(-\cos ^{2} \frac{\pi}{k}\right)\right)\left(\beta \neq \arccos \left(-\cos ^{2} \frac{\pi}{k}\right)\right.$, otherwise $T$ would be isosceles $)$. The corresponding 3D representations for $3 \leq k \leq 5$ are illustrated in Figure 53. We denote such family of f-tilings by $\mathcal{R}_{\delta \beta}^{k}$, with $k \geq 3$ and $\beta+\delta \in\left(\frac{(k-1) \pi}{k}, \frac{\pi}{k}+\arccos \left(-\cos ^{2} \frac{\pi}{k}\right)\right)$, $\delta<\frac{\pi}{k}<\beta$.

(ii) If $\alpha_{2}+\beta<\pi$, then $\theta_{4}=\alpha_{2}$ or $\theta_{4}=\delta$ (see Figure 54(a)). In both cases we reach a contradiction, as we can observe in Figure 54(b) and Figure 55, respectively, where we have no way to avoid an incompatibility between sides at vertex $v$.

2.3 If $\theta_{3}=\gamma$ (Figure 50(a)), then necessarily $\beta+\gamma=\pi$ and we obtain the configuration 


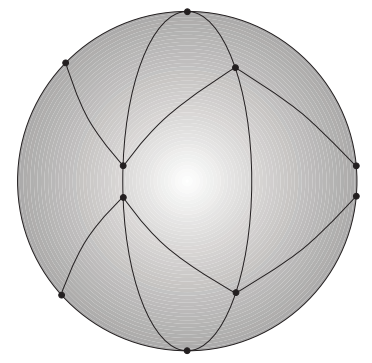

(a) $k=3$

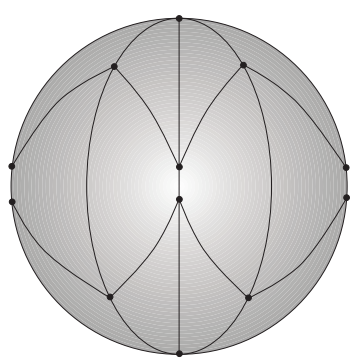

(b) $k=4$

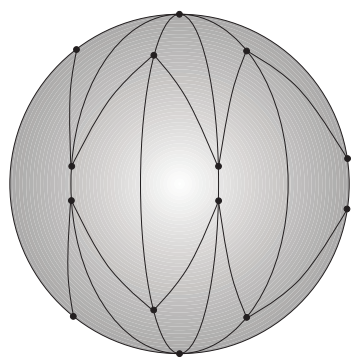

(c) $k=5$

Figure 53: 3D representations of $\mathcal{R}_{\delta \beta}^{k}, k \geq 3$ and $\beta+\delta \in\left(\frac{(k-1) \pi}{k}, \frac{\pi}{k}+\arccos \left(-\cos ^{2} \frac{\pi}{k}\right)\right)$

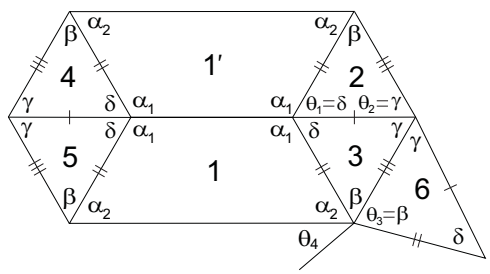

(a)

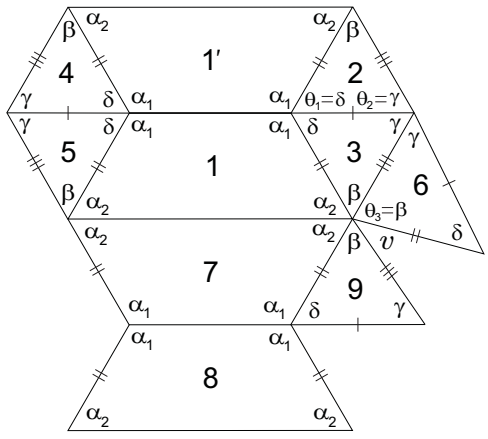

(b)

Figure 54: Local configurations

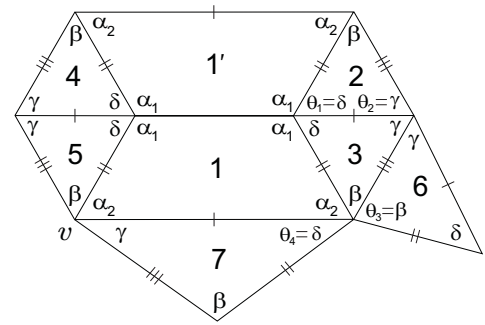

(a)

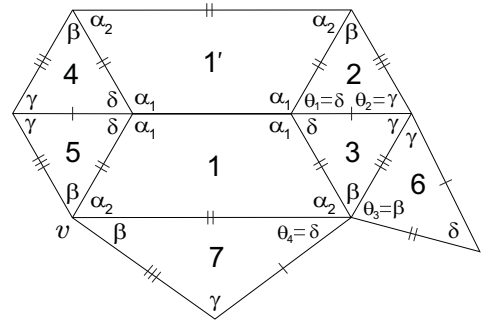

(b)

Figure 55: Local configurations

illustrated in Figure 56(a). Now, if $\alpha_{2}+\gamma=\pi$, and consequently $\alpha_{1}>\alpha_{2}=\beta>\gamma>\delta$, we get the configuration illustrated in Figure 56(b). Nevertheless, a contradiction is achieved at vertex $v_{3}$. On the other hand, if $\alpha_{2}+\gamma<\pi$, we have necessarily $\theta_{4}=\alpha_{2}$ or $\theta_{4}=\delta$. In both cases we reach a contradiction as it can be seen in Figure 57 ((a) and (b)), where we have no way to avoid an incompatibility between sides at vertex $v$.

Proposition 2.4 There are no tilings with two quadrangles in adjacent positions as presented in Figure 3, with $\theta_{1}=\gamma$ and $\alpha_{1}+\gamma<\pi$. 


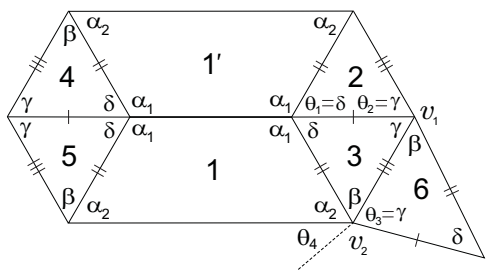

(a)

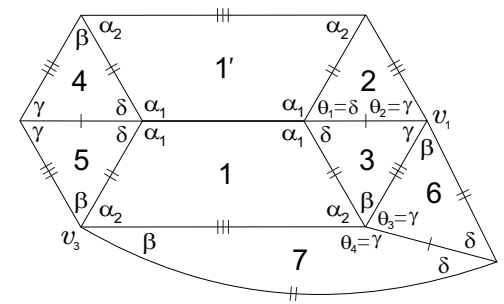

(b)

Figure 56: Local configurations

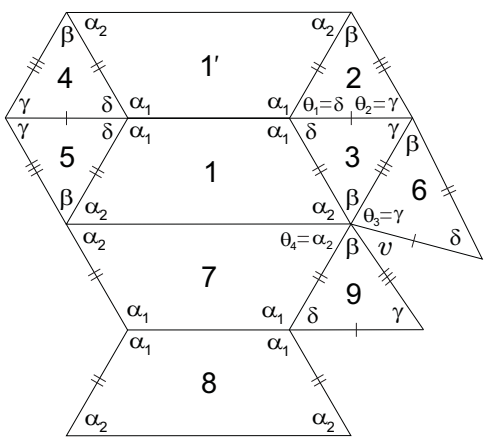

(a)

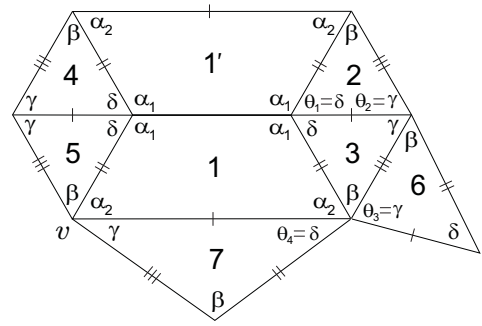

(b)

Figure 57: Local configurations

Proof. Suppose that we have two cells in adjacent positions as illustrated in Figure 58(a), with $\alpha_{1}+\gamma<\pi$. Therefore $\beta>\alpha_{1}>\alpha_{2}>\gamma>\delta$ and, with the labelling used in this figure, we have

$$
\theta_{2}=\beta \quad \text { or } \quad \theta_{2}=\delta .
$$

1. If $\theta_{2}=\beta$ (Figure 58(b)), then we must have $\theta_{3}=\gamma$ (see edge lengths) and we get the

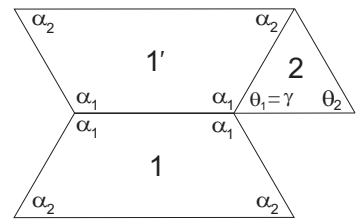

(a)

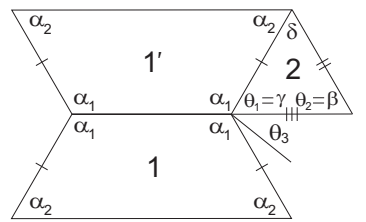

(b)

Figure 58: Local configurations

local configuration illustrated in Figure 59(a). Now, taking into account the edge lengths, we must have $\theta_{4}=\delta$. Consequently, $\theta_{5}=\beta$ and we reach a contradiction at vertex $v$.

2. If $\theta_{2}=\delta$ (Figure 59(b)), then we have $\theta_{3}=\gamma$ or $\theta_{3}=\delta$.

If $\theta_{3}=\gamma$, then $\beta+\gamma=\pi$, and $\theta_{4}=\delta$ or $\theta_{4}=\beta$. In both cases, as we can observe in Figure 60 , we reach a contradiction (there is no way to satisfy the angle-folding relation 


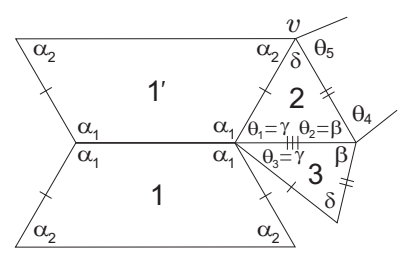

(a)

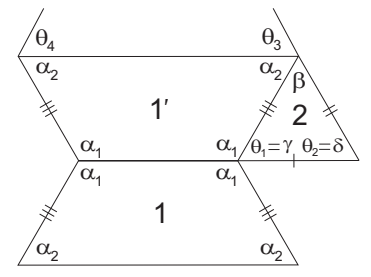

(b)

Figure 59: Local configurations

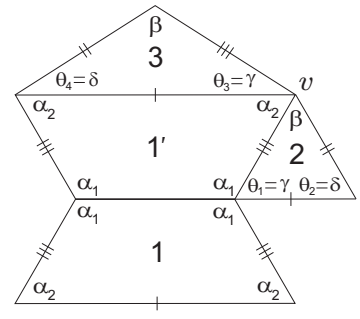

(a)

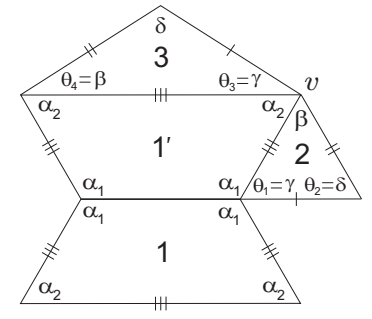

(b)

Figure 60: Local configurations

around vertex $v$ ).

If $\theta_{3}=\delta$ and $\beta+\delta=\pi$, we also reach a contradiction (see Figure 61(a) and Figure $61(\mathrm{~b})$, respectively).

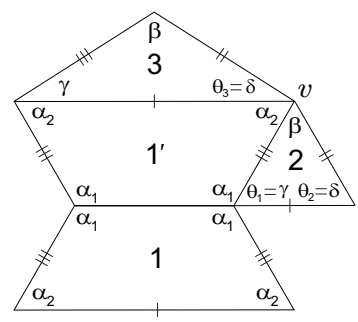

(a)

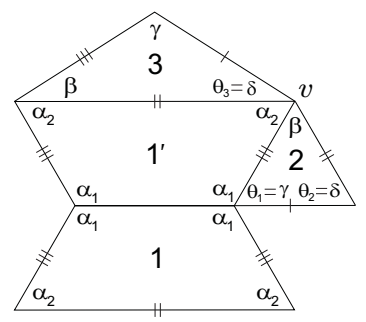

(b)

Figure 61: Local configurations

If $\theta_{3}=\delta$ and $\beta+\delta<\pi$, then $\beta+k \delta=\pi$, for some $k \geq 2$. Now, if $\theta_{4}=\gamma$ (Figure 62(a)), we have no way to avoid an incompatibility between sides at vertex $v$. On the other hand, if $\theta_{4}=\beta$ (Figure $62(\mathrm{~b})$ ), we obtain $\beta+k \delta=\pi=\alpha_{2}+k \delta$, which is a contradiction (note that $\left.\alpha_{2} \neq \beta\right)$.

Proposition 2.5 If $\theta_{1}=\gamma$ and $\alpha_{1}+\gamma=\pi$ (Figure 3), then $\Omega(Q, T) \neq \emptyset$ iff

(i) $\alpha_{2}=\beta=\frac{\pi}{2}$ and $\delta=\frac{\pi}{2 k}, k \geq 2$, or 


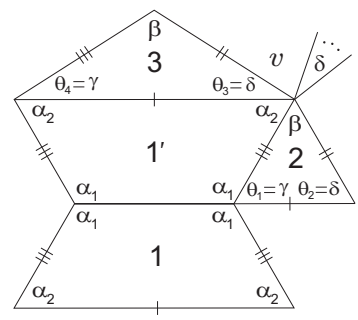

(a)

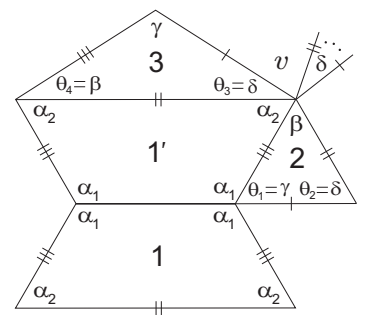

(b)

Figure 62: Local configurations

(ii) $\alpha_{2}+\beta=\pi$ and $\delta=\frac{\pi}{k}, k \geq 3$, or

(iii) $\beta=\frac{\pi}{2}, \gamma=\frac{\pi}{3}$ and $\alpha_{2}+2 \delta=\pi$, or

(iv) $\beta=\frac{\pi}{2}, \gamma=\frac{\pi}{3}, \delta=\frac{\pi}{5}$ and $\alpha_{2}=\frac{2 \pi}{5}$.

In the first case, for each $k \geq 2$, we obtain a single tiling, denoted by $\mathcal{T}^{k}$, with $\gamma=$ $\arccos \frac{\sin \frac{\pi}{2 k}}{2}$. A planar representation of $\mathcal{T}^{k}$ is given in Figure 66 and $3 D$ representations, for $k=2$ and $k=3$, are given in Figure 67 .

The case (ii) leads to a family of f-tilings, $\mathcal{R}_{\beta \gamma}^{k}$, with $\frac{(k-1) \pi}{k}<\gamma+\beta<\pi$ where $\beta \neq$ $\arccos \left(-\cos ^{2} \frac{\pi}{k}\right)$. A planar representation of $\mathcal{R}_{\beta \gamma}^{k}$ is given in Figure 72(b) and 3D representations, for $k=3, k=4$ and $k=5$, are given in Figure 73.

The case (iii) leads to a family of f-tilings, $\mathcal{M}_{\delta}^{3}$, with $\frac{\pi}{6}<\delta<\frac{1}{2} \arccos \left(-\frac{1}{4}\right)$. Planar and $3 D$ representations are given in Figure 81(b) and Figure 82, respectively.

In the last situation, we obtain a sporadic tiling, denoted by $\mathcal{G}$. Figure 83 and Figure 84 illustrate planar and $3 D$ representations of $\mathcal{G}$, respectively.

For each tiling, the angles around vertices are positioned as illustrated in Figure 63.

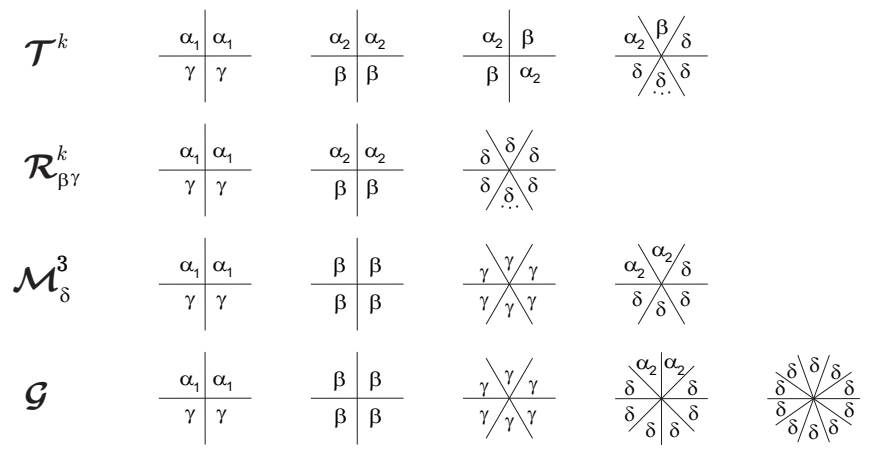

Figure 63: Distinct classes of congruent vertices

Proof. Suppose that we have two cells in adjacent positions as illustrated in Figure 3 and consider $\theta_{1}=\gamma$, with $\alpha_{1}+\gamma=\pi$. We have $\alpha_{1}>\alpha_{2}>\gamma>\delta$ and, with the labelling of 
Figure 64(a), we have

$$
\theta_{2}=\delta \quad \text { or } \quad \theta_{2}=\beta
$$

1. Suppose firstly that $\theta_{2}=\delta$. With the labelling used in Figure 64(b), we have

$$
\theta_{3}=\alpha_{2}, \quad \theta_{3}=\delta \quad \text { or } \quad \theta_{3}=\beta
$$

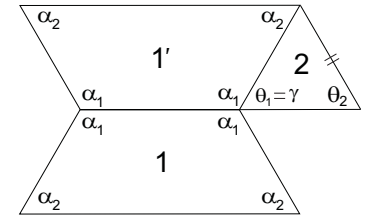

(a)

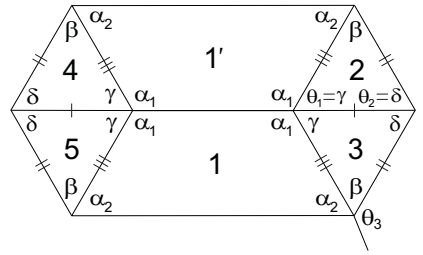

(b)

Figure 64: Local configurations

1.1 Suppose that $\theta_{3}=\alpha_{2}$ (Figure 65(a)). Taking into account the angle's relations and the edge lengths, it can be proved that $\theta_{4}=\beta$. Moreover, vertex $v$ has valency four, with $\alpha_{2}=\beta=\frac{\pi}{2}$, and we get the configuration illustrated in Figure 65(b). At vertex $v$ we

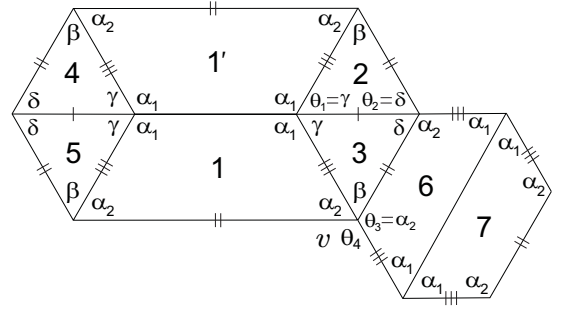

(a)

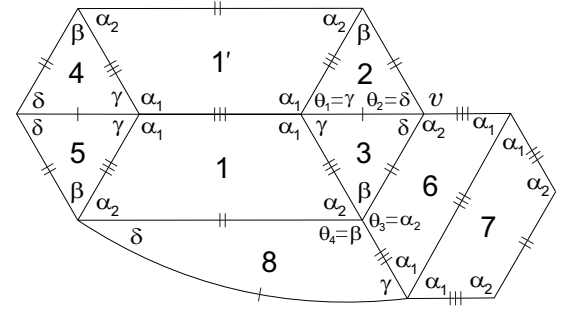

(b)

Figure 65: Local configurations

have $\frac{\pi}{2}+k \delta=\pi$, for some $k \geq 2$, i.e., $\delta=\frac{\pi}{2 k}$. The last configuration is then extended in a unique way to the one illustrated in Figure 66.

The vertices $v_{1}$ and $v_{2}$ in Figure 66 are in antipodal positions since there exist two distinct geodesics of the same length joining them, and so $b=e=\frac{\pi}{3}$. We have $\alpha_{1}=\pi-\gamma$, $\alpha_{2}=\beta=\frac{\pi}{2}$ and $\delta=\frac{\pi}{2 k}$, for some $k \geq 2$. Using these relations, it is a straightforward exercise to show that $\gamma=\arccos \frac{\sin \frac{\pi}{2 k}}{2}$. We shall denote such family of dihedral f-tilings by $\mathcal{T}^{k}, k \geq 2$. 3D representations of $\mathcal{T}^{2}$ and $\mathcal{T}^{3}$ are illustrated in Figure 67.

1.2 Suppose now that $\theta_{3}=\delta$ (Figure 68(a); recall that $\alpha_{1}>\alpha_{2}>\gamma>\delta$ ). We have $\alpha_{2}+\delta<\alpha_{1}+\gamma=\pi$. Thus, vertex $v$ has valency greater than four. As $\alpha_{2}>\gamma$ and $\beta+\gamma+\delta>\pi$, the alternating angle sum containing $\beta$ must be $\beta+k \delta=\pi$, for some $k \geq 2$. Taking into account the edge lengths, it results that the alternating angle sum 


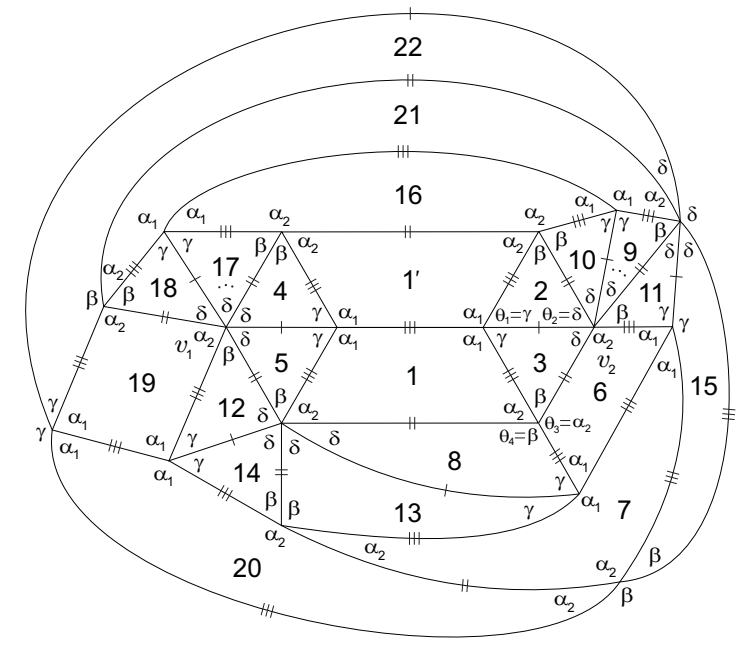

Figure 66: Planar representation of $\mathcal{T}^{k}$

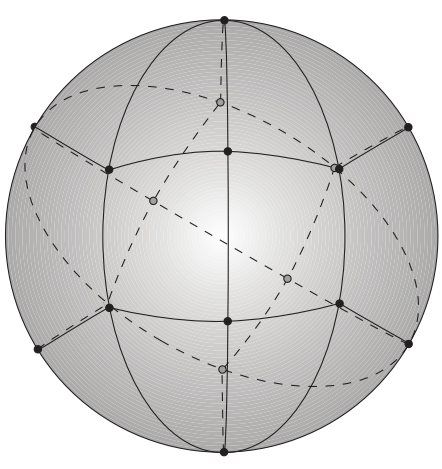

(a) $k=2$

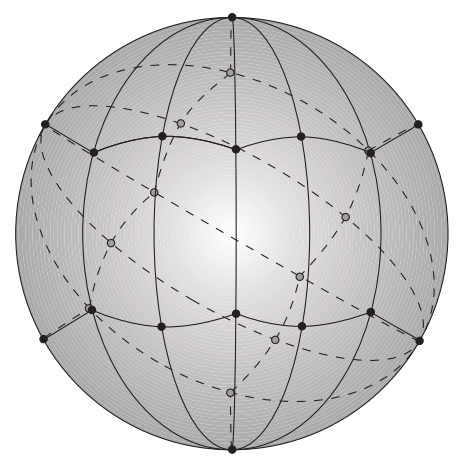

(b) $k=3$

Figure 67: 3D representations of $\mathcal{T}^{k}$

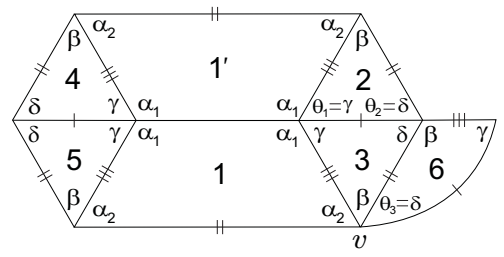

(a)

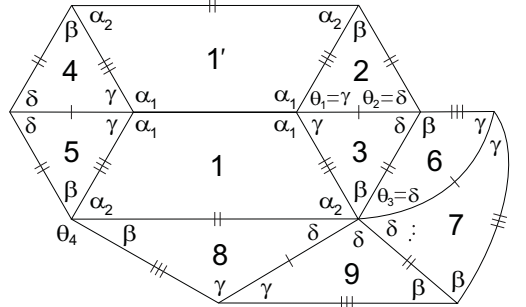

(b)

Figure 68: Local configurations

containing $\alpha_{2}$ at vertex $v$ must be $\alpha_{2}+\delta+(k-1) \delta=\alpha_{2}+k \delta=\pi$, and so $\alpha_{2}=\beta$. The last configuration is then extended to the one illustrated in Figure 68(b). Now, we have $\theta_{4} \in\left\{\alpha_{2}, \beta\right\}$. We only consider $\theta_{4}=\beta$, otherwise, by symmetry, we obtain the previous f-tilings $\mathcal{T}^{k}, k \geq 2$. Then $\beta=\alpha_{2}=\frac{\pi}{2}$ and we get the configuration illustrated in 
Figure 69(a).

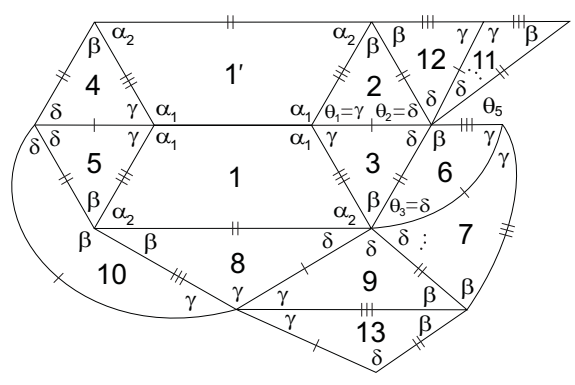

(a)

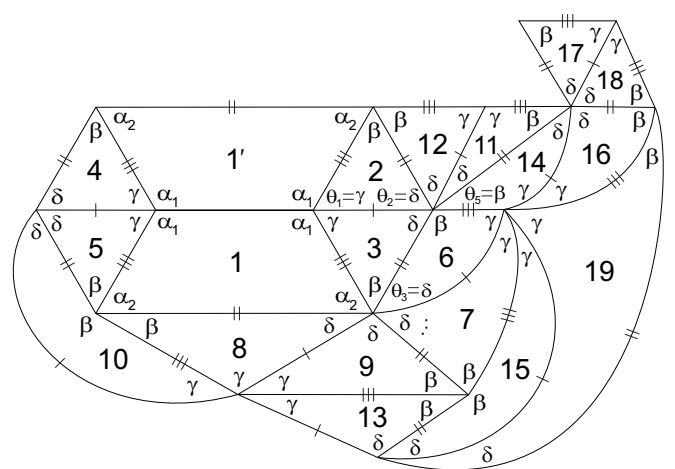

(b)

Figure 69: Local configurations

Now, $\theta_{5}$ must be $\beta$ or $\alpha_{2}$. If $\theta_{5}=\beta$, then $3 \gamma=\pi$, i.e., $\gamma=\frac{\pi}{3}$, and so $\alpha_{1}=\frac{2 \pi}{3}$, $\alpha_{2}=\beta=\frac{\pi}{2}, \gamma=\frac{\pi}{3}$ and $\delta=\frac{\pi}{4}$. We get the configuration illustrated in Figure 69(b), and consequently the decomposition in Figure 70. As $T$ and $T^{\prime}$ have one side in common

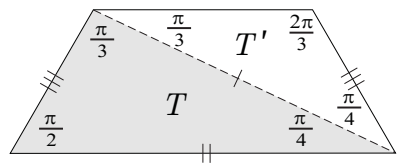

Figure 70: $Q=T \cup T^{\prime}$

and the adjacent angles are equal, we conclude that $T$ and $T^{\prime}$ are congruent, which is an impossibility.

On the other hand, if $\theta_{5}=\alpha_{2}$, we get the configuration illustrated in Figure 71(a). Now, at vertex $v$ we have $\gamma+\gamma<\pi$. As $\beta=\frac{\pi}{2}$, we obtain $\gamma+\gamma+\gamma=\pi$ or $\gamma+\gamma+\delta=\pi$ (recall that $\gamma+\delta>\frac{\pi}{2}$ ). But, if we have $\gamma=\frac{\pi}{3}$, then $\delta=\frac{\pi}{4}$ and we reach a contradiction as previously (see Figure 70). Therefore, $\gamma+\gamma+\delta=\pi$ (Figure 71(b)), and, at vertex $v$, it results that $\alpha_{2}+\gamma<\pi$ and $\alpha_{2}+\gamma+\rho>\pi, \forall \rho \in\left\{\alpha_{1}, \alpha_{2}, \beta, \gamma, \delta\right\}$, which is a contradiction.

1.3 Suppose finally that $\theta_{3}=\beta$. Analogously to the case 1.1 , we have $x_{i} \in\left\{\alpha_{2}, \beta, \delta\right\}$, $i=1,2,3$ (Figure 72(a)). It is enough to consider $x_{i}=\beta, \forall i$, otherwise we obtain, by symmetry, the f-tilings $\mathcal{T}^{k}, k \geq 2$, or an absurdity. As a trapezoid must always have an adjacent trapezoid sharing the side of length $c$, the last configuration is then extended to the one illustrated in Figure 72(b). A complete planar representation is uniquely determined, where $\alpha_{1}+\gamma=\pi, \alpha_{2}+\beta=\pi, \delta=\frac{\pi}{k}$, for some $k \geq 3$, with $\alpha_{1}>\alpha_{2}>\gamma$ and $\alpha_{1}>\beta>\gamma$.

We denote such family of f-tilings by $\mathcal{R}_{\beta \gamma}^{k}$, with $k \geq 3$ and $\beta+\gamma \in\left(\frac{(k-1) \pi}{k}, \pi\right), \gamma>\frac{\pi}{k}$. The corresponding 3D representations for $k=3, k=4$ and $k=5$ are given in Figure 73 . 


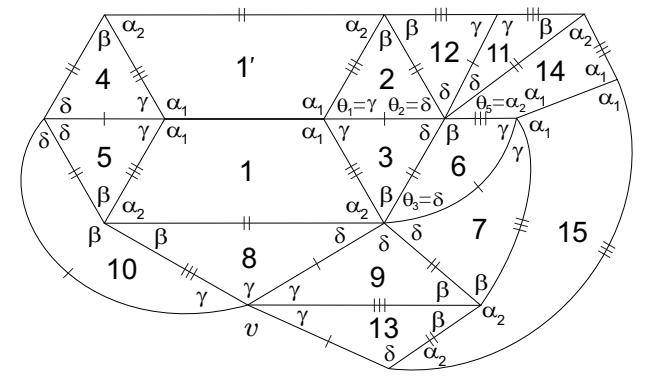

(a)

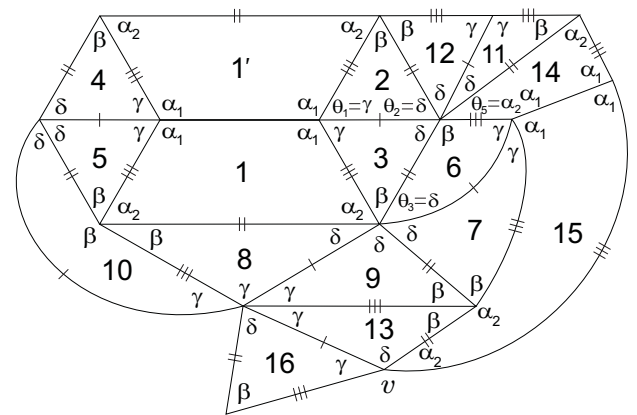

(b)

Figure 71: Local configurations

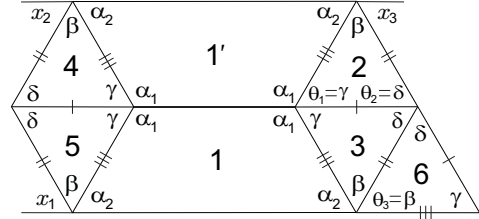

(a)

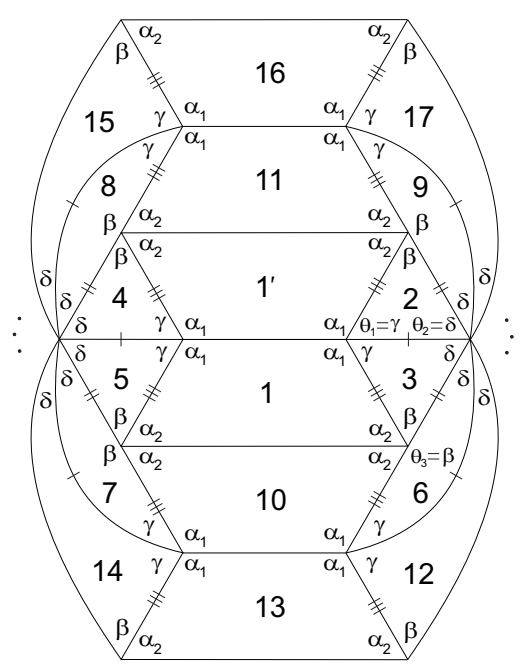

(b) Planar representation of $\mathcal{R}_{\beta \gamma}^{k}$

Figure 72: Local configurations

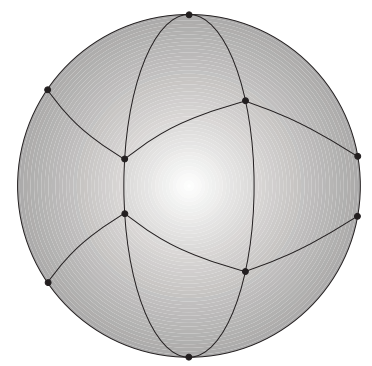

(a) $k=3$

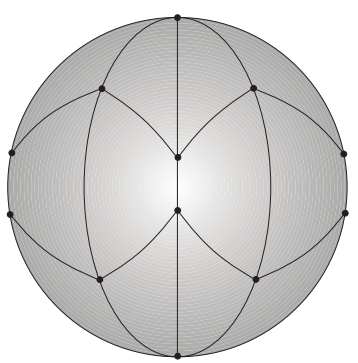

(b) $k=4$

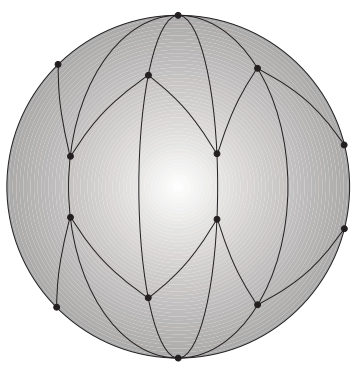

(c) $k=5$

Figure 73: 3D representations of $\mathcal{R}_{\beta \gamma}^{k}, k=3,4,5, \beta+\gamma \in\left(\frac{(k-1) \pi}{k}, \pi\right), \gamma>\frac{\pi}{k}$ 
2. Suppose now that $\theta_{2}=\beta$ (Figure 64(a)). Recall that $\alpha_{1}>\alpha_{2}>\gamma>\delta$. With the labelling used in Figure 74(a), we have

$$
\theta_{3}=\alpha_{2}, \quad \theta_{3}=\beta \quad \text { or } \quad \theta_{3}=\delta .
$$

2.1 If $\theta_{3}=\alpha_{2}$, then we get the local configuration illustrated in Figure 74(b), with

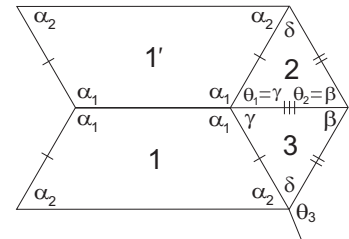

(a)

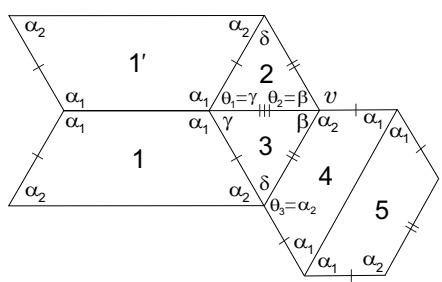

(b)

Figure 74: Local configurations

$\alpha_{2}+\beta=\pi$. We reach a contradiction at vertex $v$ since there is no way to satisfy the angle-folding relation around this vertex.

2.2 If $\theta_{3}=\beta$, we obtain the local configuration illustrated in Figure 75(a). At vertex $v$

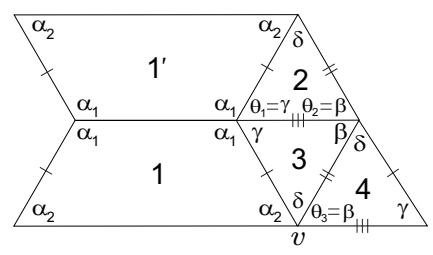

(a)

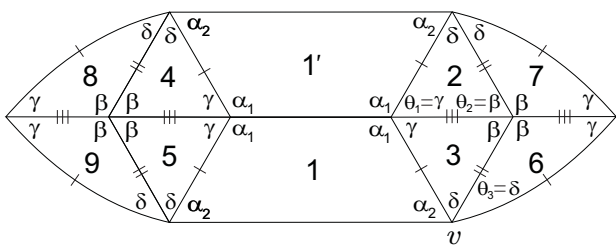

(b)

Figure 75: Local configurations

we must have $\alpha_{2}+\beta=\pi$. Nevertheless, $\delta+\rho<\pi, \forall \rho \in\left\{\alpha_{1}, \alpha_{2}, \beta, \gamma, \delta\right\}$, which is an incongruity.

2.3 Finally, if we consider $\theta_{3}=\delta$, then the last configuration is extended to get the one illustrated in Figure $75(\mathrm{~b})$. As $\beta=\frac{\pi}{2}, \alpha_{2}>\gamma$ and $\gamma+\delta>\frac{\pi}{2}$, we must have, at vertex $v$,

$$
\alpha_{2}+\delta+\gamma=\pi, \quad \alpha_{2}+\delta+\alpha_{2}=\pi \quad \text { or } \quad \alpha_{2}+k \delta=\pi, \text { for some } k \geq 2 .
$$

2.3.1 Suppose firstly that $\alpha_{2}+\delta+\gamma=\pi$. If $\theta_{4}=\beta$ (Figure 76(a)), then we have necessarily $\theta_{5}=\alpha_{2}$ or $\theta_{5}=\gamma$. In the first case, we obtain the configuration illustrated in Figure 76(b). At vertex $v$ we must have $\alpha_{1}+k \delta=\pi$, for some $k \geq 2$, which is a contradiction, by Proposition 2.2. In the last case $\left(\theta_{5}=\gamma\right)$, we also reach a contradiction at vertex $v$, see Figure $77(\mathrm{a})$. 


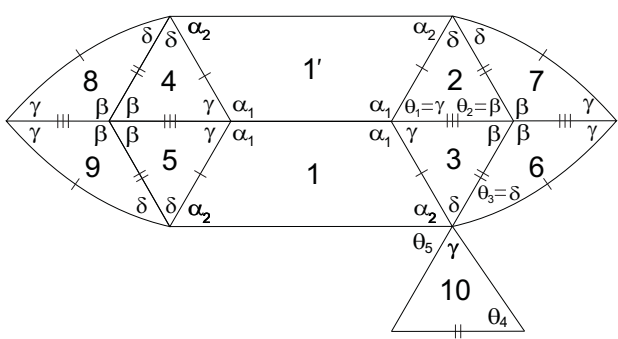

(a)

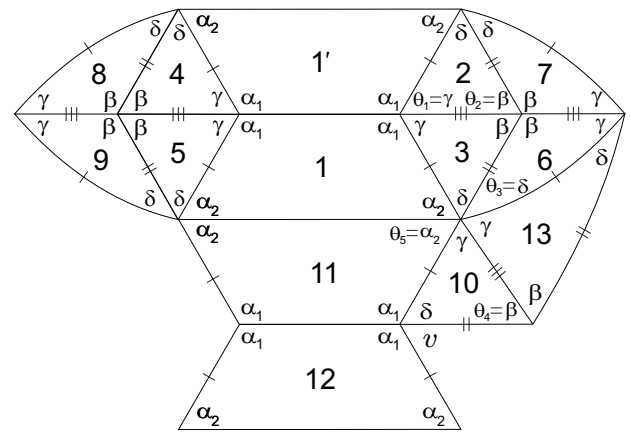

(b)

Figure 76: Local configurations

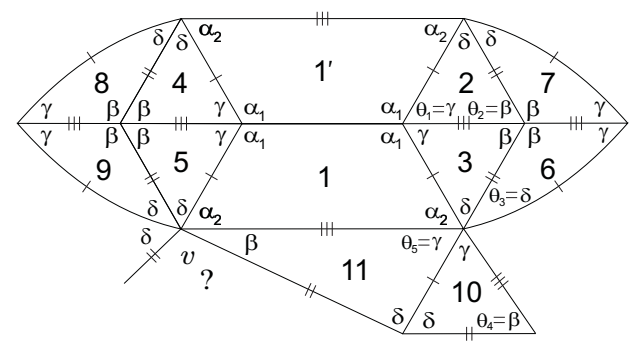

(a)

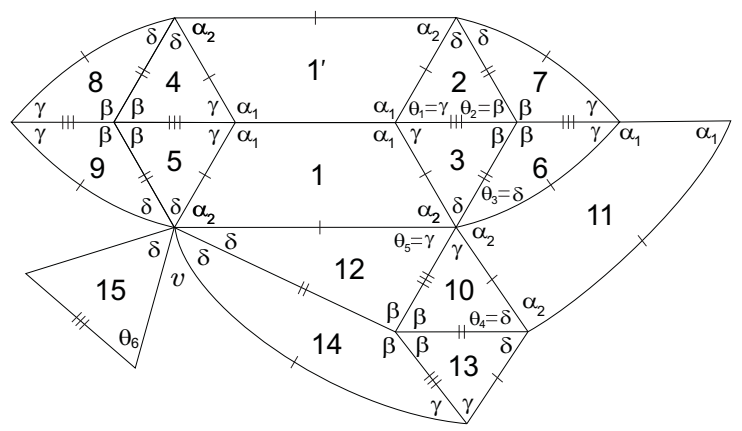

(b)

Figure 77: Local configurations

If $\theta_{4}=\delta$ (Figure 76(a)), we obtain the configuration illustrated in Figure 77(b). At vertex $v$ we must have $\alpha_{2}+k \delta=\pi$, for some $k \geq 3$. Now, the distinct cases $\theta_{6}=\gamma$ and $\theta_{6}=\beta$ are similar, so we only consider $\theta_{6}=\gamma$. This condition gives rise to the configuration illustrated in Figure 78(a). Note that the conditions $\gamma+\gamma+\delta<\pi(=$ $\left.\alpha_{2}+\gamma+\delta\right), \gamma+\gamma+\alpha_{2}>\pi$ and $\gamma+\gamma+2 \delta>\pi$ imply $\gamma=\frac{\pi}{3}$. Thus, $\gamma=(k-1) \delta$, and so $\delta \leq \frac{\pi}{6}$. But then $\beta+\gamma+\delta \leq \pi$, which is an impossibility.

2.3.2 If $\alpha_{2}+\delta+\alpha_{2}=\pi$ we obtain the configuration illustrated in Figure 78(b), and 


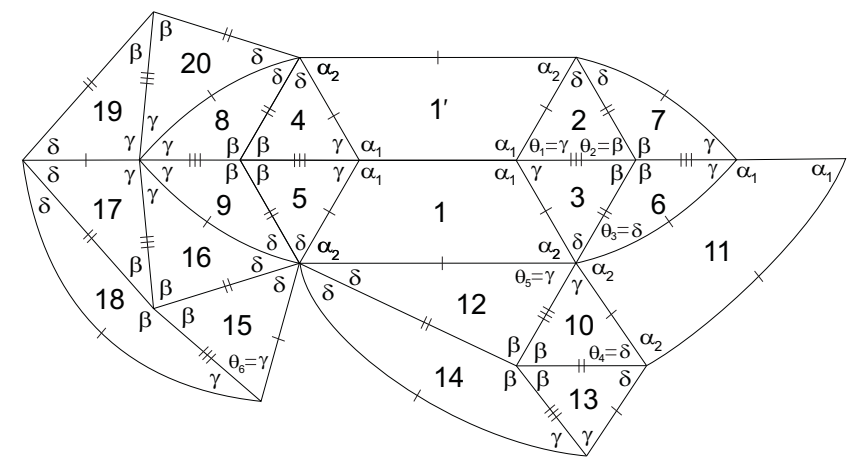

(a)

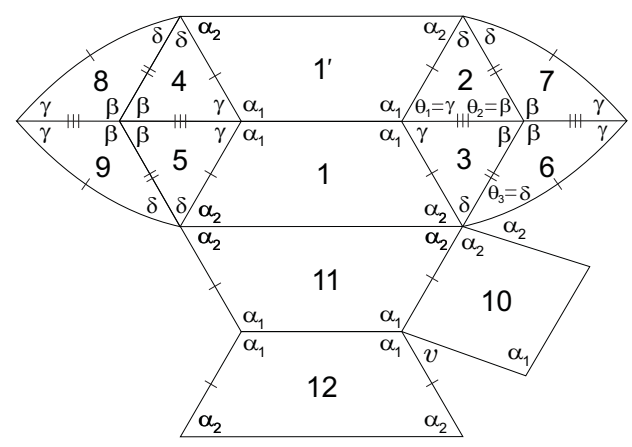

(b)

Figure 78: Local configurations

consequently a contradiction at vertex $v$.

2.3.3 Finally, we consider the case $\alpha_{2}+k \delta=\pi$, for some $k \geq 2$. With the labelling used in Figure 79(a), we have $\theta_{4}=\beta, \theta_{4}=\gamma, \theta_{4}=\delta$ or $\theta_{4}=\alpha_{2}$.

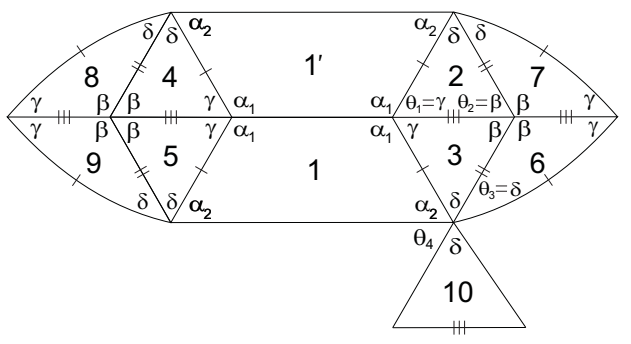

(a)

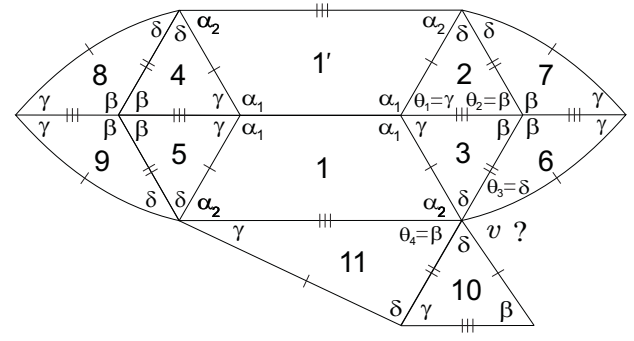

(b)

Figure 79: Local configurations

The first three cases lead to contradictions. In fact, if $\theta_{4}=\beta$, see Figure $79(\mathrm{~b})$, at vertex $v$ we must have $\alpha_{2}+k \delta=\pi=\beta+k \delta$, for some $k \geq 2$. Nevertheless, there is no way to avoid an incompatibility between sides at this vertex. If $\theta_{4}=\gamma$ (Figure 80(a)), at vertex $v$ we get $\beta+\gamma<2 \beta=\pi$ and $\beta+\gamma+\rho>\pi, \forall \rho \in\left\{\alpha_{1}, \alpha_{2}, \beta, \gamma, \delta\right\}$. In the case $\theta_{4}=\delta$ (Figure $80(\mathrm{~b})$ and Figure $81(\mathrm{a})$ ) there is no way to avoid an incompatibility between sides at vertex $v$.

Finally, we shall consider $\theta_{4}=\alpha_{2}$ (Figure 79(a)). We obtain $\gamma=\frac{\pi}{3}$, and so $\delta>\frac{\pi}{6}$. Now, as $\alpha_{2}>\gamma$ and $\alpha_{2}+k \delta=\pi$, it follows that $k=2$ or $k=3$. If $k=2$, the configuration is extended in a unique way to the one given in Figure 81(b). Using spherical trigonometry formulas, it can be seen that $\delta \in\left(\frac{\pi}{6}, \frac{1}{2} \arccos \left(-\frac{1}{4}\right)\right)$. We shall denote such f-tilings by $\mathcal{M}_{\delta}^{3}$. Its 3D representation is shown in Figure 82.

When $k=3$, we obtain the planar representation given in Figure 83. It results that $t \delta=\pi$, where $t=4$ or $t=5$. As $\delta=\frac{\pi}{4}$ and $\alpha_{2}+3 \delta=\pi$ imply $\alpha_{2}=\frac{\pi}{4}$, which is a contradiction, we conclude that $t=5$. And so $\alpha_{1}=\frac{2 \pi}{3}, \alpha_{2}=\frac{2 \pi}{5}, \beta=\frac{\pi}{2}, \gamma=\frac{\pi}{3}$ and $\delta=\frac{\pi}{5}$. We shall denote such f-tiling by $\mathcal{G}$. Its $3 \mathrm{D}$ representation is shown in Figure 84. 


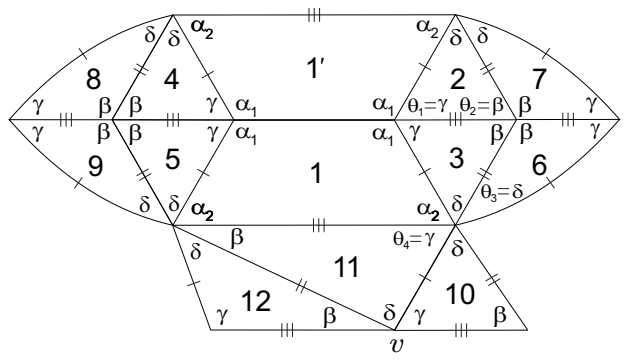

(a)

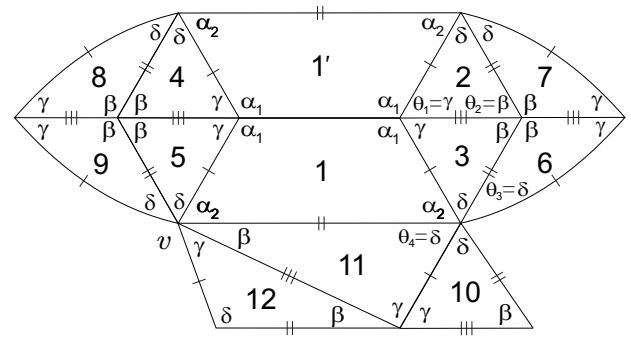

(b)

Figure 80: Local configurations

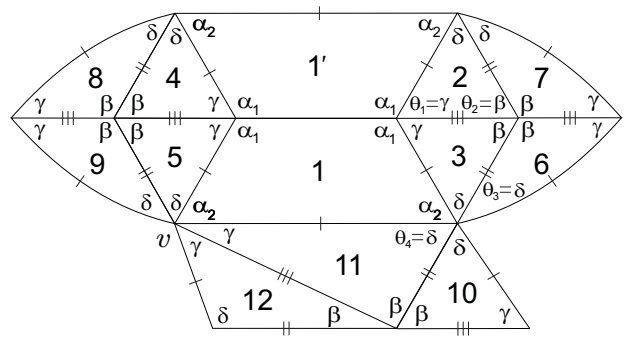

(a)

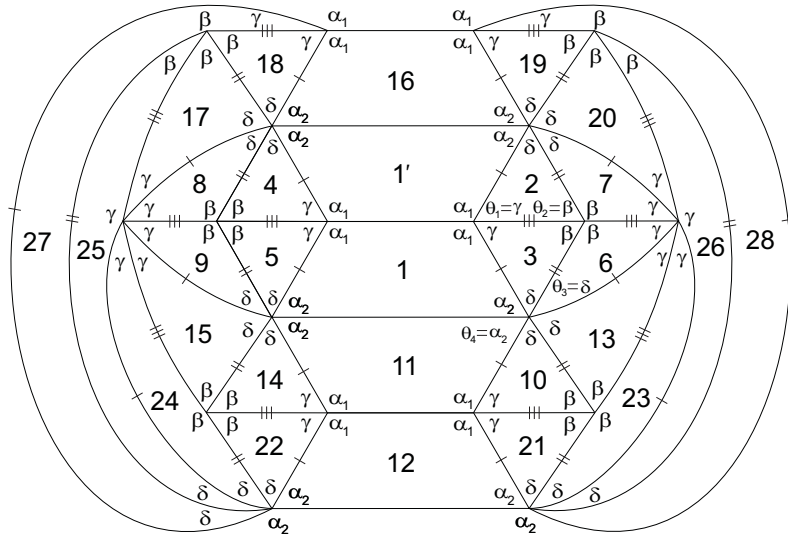

(b) Planar representation of $\mathcal{M}_{\delta}^{3}$

Figure 81: Local configurations

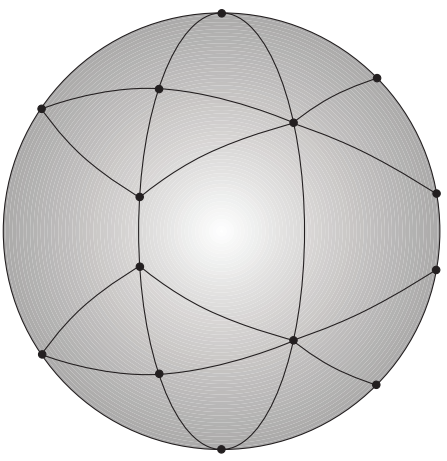

Figure 82: 3D representation of $\mathcal{M}_{\delta}^{3}, \delta \in\left(\frac{\pi}{6}, \frac{1}{2} \arccos \left(-\frac{1}{4}\right)\right)$

\section{Summary}

In Table 1 is shown a list of the spherical dihedral f-tilings whose prototiles are a spherical isosceles trapezoid $Q$ of internal angles $\alpha_{1}$ and $\alpha_{2}\left(\alpha_{1}>\alpha_{2}\right)$ and an scalene triangle $T$ of angles $\beta, \gamma$ and $\delta(\beta>\gamma>\delta)$ obtained in this paper. Our notation is as follows: 


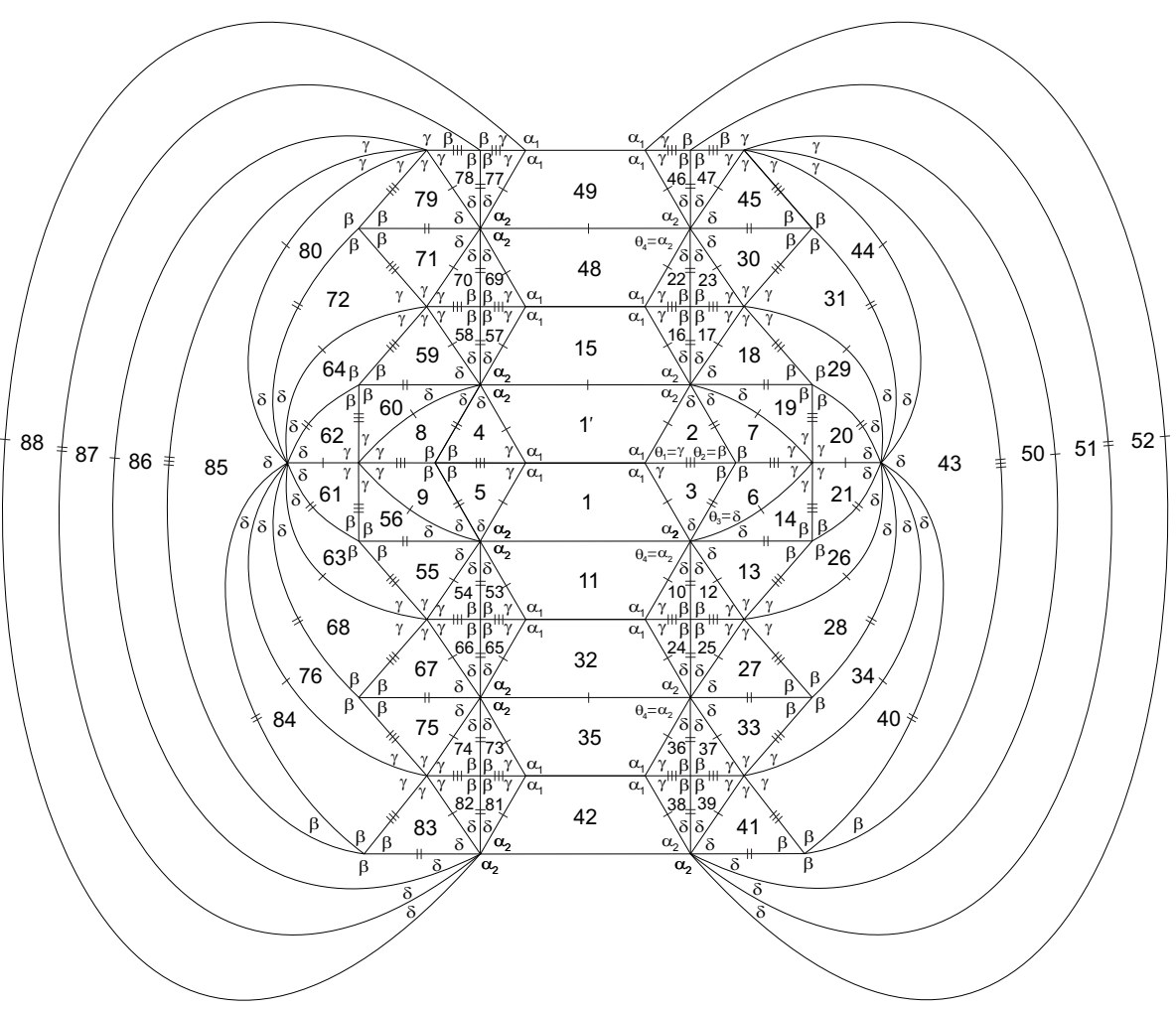

Figure 83: Planar representation of $\mathcal{G}$

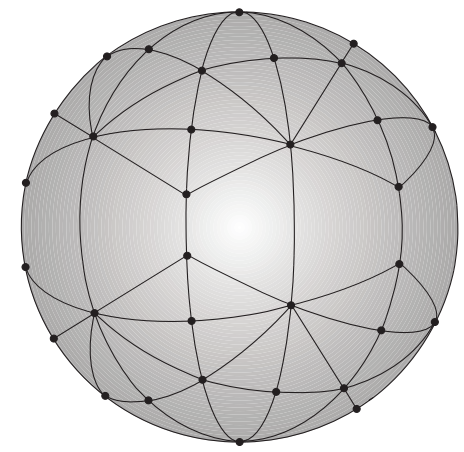

Figure 84: 3D representation of $\mathcal{G}$

- $\phi(k)=\frac{1}{2} \arccos \left(-\cos ^{2} \frac{\pi}{k}\right)$;

- $|V|$ is the number of distinct classes of congruent vertices;

- $M$ and $N$ are, respectively, the number of triangles congruent to $T$ and the number of isosceles trapezoids congruent to $Q$, used in the dihedral f-tilings.

- $G(\tau)$ is the symmetry group of each tiling $\tau \in \Omega(Q, T)$; by $D_{n}$ we mean the dihedral group of order $2 n ; V$ is the Klein 4-group. 


\begin{tabular}{|c|c|c|c|c|c|c|c|c|c|}
\hline F-Tiling & $\boldsymbol{\alpha}_{\mathbf{1}}$ & $\boldsymbol{\alpha}_{\mathbf{2}}$ & $\boldsymbol{\beta}$ & $\boldsymbol{\gamma}$ & $\boldsymbol{\delta}$ & $|V|$ & $M$ & $N$ & $G(\tau)$ \\
\hline \hline $\boldsymbol{\mathcal { R }}_{\boldsymbol{\delta} \boldsymbol{\gamma}}^{\mathbf{2}}$ & $\pi-\delta$ & $\pi-\gamma$ & $\frac{\pi}{2}$ & $\gamma$ & $\delta$ & 3 & 8 & 4 & $D_{4}$ \\
\hline $\boldsymbol{\mathcal { R }}_{\boldsymbol{\delta} \boldsymbol{\beta}}^{\boldsymbol{k}}, \boldsymbol{k} \geq \mathbf{3}$ & $\pi-\delta$ & $\pi-\beta$ & $\beta$ & $\frac{\pi}{k}$ & $\delta$ & 3 & $4 \mathrm{k}$ & $2 \mathrm{k}$ & $D_{2 k}$ \\
\hline $\boldsymbol{\mathcal { R }}_{\boldsymbol{\beta} \boldsymbol{\gamma}}^{\boldsymbol{r}}, \boldsymbol{k} \geq \mathbf{3}$ & $\pi-\gamma$ & $\pi-\beta$ & $\beta$ & $\gamma$ & $\frac{\pi}{k}$ & 3 & $4 \mathrm{k}$ & $2 \mathrm{k}$ & $D_{2 k}$ \\
\hline $\mathcal{M}_{\boldsymbol{\delta}}^{\mathbf{3}}$ & $\pi-\gamma$ & $\pi-2 \delta$ & $\frac{\pi}{2}$ & $\frac{\pi}{3}$ & $\left(\frac{\pi}{6}, \phi(3)\right)$ & 4 & 24 & 6 & $D_{6}$ \\
\hline $\mathcal{M}_{\boldsymbol{\gamma}}^{\boldsymbol{k}}, \boldsymbol{k} \geq \mathbf{4}$ & $\pi-\delta$ & $\pi-2 \gamma$ & $\frac{\pi}{2}$ & $\left(\frac{(k-2) \pi}{2 k}, \phi(k)\right)$ & $\frac{\pi}{k}$ & 4 & $8 \mathrm{k}$ & $4 \mathrm{k}$ & $D_{2 k}$ \\
\hline $\mathcal{T}^{\boldsymbol{k}}, \boldsymbol{k} \geq \mathbf{2}$ & $\pi-\gamma$ & $\frac{\pi}{2}$ & $\frac{\pi}{2}$ & $\arccos \frac{\sin \frac{\pi}{2 k}}{2}$ & $\frac{\pi}{2 k}$ & 4 & $8 k$ & $4 k$ & $V$ \\
\hline $\mathcal{N}$ & $\frac{3 \pi}{5}$ & $\frac{\pi}{2}$ & $\frac{\pi}{2}$ & $\frac{\pi}{3}$ & $\frac{\pi}{5}$ & 5 & 60 & 10 & $D_{10}$ \\
\hline $\mathcal{G}$ & $\frac{2 \pi}{3}$ & $\frac{2 \pi}{5}$ & $\frac{\pi}{2}$ & $\frac{\pi}{3}$ & $\frac{\pi}{5}$ & 5 & 80 & 10 & $D_{10}$ \\
\hline
\end{tabular}

Table 1: Combinatorial Structure of the Dihedral F-Tilings of $S^{2}$ by Isosceles Trapezoids and Scalene Triangles with adjacency of type V (Figure 2)

\section{References}

[1] A. M. Breda and A. F. Santos, Dihedral f-tilings of the sphere by spherical triangles and equiangular well-centered quadrangles, Beiträge zur Algebra und Geometrie, 45 (2004), 447-461.

[2] A. M. Breda and A. F. Santos, Dihedral f-tilings of the sphere by rhombi and triangles, Discrete Math. Theoretical Computer Sci., 7 (2005), 123-140.

[3] A. M. Breda and A. F. Santos, Dihedral f-tilings of the sphere by triangles and well-centered quadrangles, Hiroshima Math. J., 36 (2006), 235-288.

[4] A. M. Breda, P. S. Ribeiro and A. F. Santos, A class of spherical dihedral f-tilings, European Journal of Combinatorics, 30(1) (2009), 119-132.

[5] C. P. Avelino and A. F. Santos, Spherical f-tilings by triangles and $r$-sided regular polygons, $r \geq 5$, The Electronic Journal of Combinatorics, 15 (2008), \#R22.

[6] C. P. Avelino and A. F. Santos, Spherical f-tilings by (equilateral and isosceles) triangles and isosceles trapezoids, 2008, submitted.

[7] C. P. Avelino and A. F. Santos, Spherical f-tilings by scalene triangles and isosceles trapezoids, I, European Journal of Combinatorics, 30(5) (2009), 1221-1244.

[8] C. P. Avelino and A. F. Santos, Spherical f-tilings by scalene triangles and isosceles trapezoids, II, submitted.

[9] S. A. Robertson, Isometric folding of Riemannian manifolds, Proceedings of the Royal Society of Edinburgh, 79 (1977), 275-284.

[10] R. J. Dawson, Tilings of the sphere with isosceles triangles, Disc. and Comp. Geom., 30 (2003), 467-487.

[11] R. J. Dawson and B. Doyle, Tilings of the sphere with right triangles I: the asymptotically right families, Electronic Journal of Combinatorics, 13 (2006), \#R48. 